Estabilidade Assintótica de Modelos Logísticos com Retardamento

\author{
Juliana Rodrigues Dionisio Pereira
}


SERVIÇO DE PÓS-GRADUAÇÃO DO ICMC-USP

Data de Depósito:

Assinatura:

\title{
Estabilidade Assintótica de Modelos Logísticos com Retardamento
}

\author{
Juliana Rodrigues Dionisio Pereira
}

Orientadora: Profa. Dra. Sueli Mieko Tanaka Aki

\begin{abstract}
Dissertação apresentada ao Instituto de Ciências Matemáticas e de Computação - ICMC-USP, como parte dos requisitos para obtenção do título de Mestre em Ciências - Matemática . EXEMPLAR DE DEFESA
\end{abstract}

USP - São Carlos

Abril de 2013 
Ficha catalográfica elaborada pela Biblioteca Prof. Achille Bassi e Seção Técnica de Informática, ICMC/USP, com os dados fornecidos pelo(a) autor(a)

Rodrigues Dionisio Pereira, Juliana
Estabilidade Assintótica de Modelos Logísticos com
Retardamento / Juliana Rodrigues Dionisio Pereira;
orientador Sueli Mieko Tanaka Aki. -- São Carlos,
2013.
$77 \mathrm{p}$.
Dissertação (Mestrado - Programa de Pós-Graduação
em Matemática) -- Instituto de Ciências Matemáticas
e de Computação, Universidade de São Paulo, 2013.
1. Estabilidade Assintótica de Modelos Logísticos
com Retardamento. I. Mieko Tanaka Aki, Sueli,
orient. II. Título.




\section{Sumário}

\begin{tabular}{lll}
\hline 1 & Preliminares & 7
\end{tabular}

$1.1 \quad$ Alguns resultados importantes $\ldots \ldots \ldots \ldots$. . . . . . . . . . . 7

1.2 Equação Diferencial Funcional com Retardo - Conceitos básicos . . . . . . 8

1.3 Resultados sobre existência, unicidade e dependência contínua $\ldots . . .10$

2 Equação autônoma com linearidade positiva $\quad 25$

2.1 Equação Logística Retardada . . . . . . . . . . . . . . . . . . 25

2.2 Resultados importantes . . . . . . . . . . . . . . . . . . 28

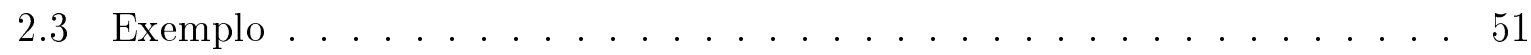

3 Equação não autônoma com linearidade positiva

3.1 Equação Logística Retardada com uma linearidade não autônoma . . . . . 53

3.2 Resultados importantes . . . . . . . . . . . . . . . . . 55

4 Equação autônoma com linearidade não positiva $\quad 67$

4.1 Equação Logística Retardada com uma linearidade autônoma não positiva 67

4.2 Resultados importantes . . . . . . . . . . . . . . . . . . 69 
4.3 Aplicação . . . . . . . . . . . . . . . . . . . . . . . 74 


\section{Agradecimentos}

Agradeço inicialmente a Deus por estar sempre ao meu lado em todos os momentos, iluminando meu caminho e me dando força e coragem para seguir sempre em frente.

Aos meus pais, Goreti e Pedrão, todo meu amor e minha gratidão pela imensa dedicação que sempre tiveram comigo e com meus irmãos, investindo todos seus esforços buscando uma formação pessoal e profissional de qualidade para seus filhos. Obrigada pelo amor incondicional, pelo exemplo de honestidade e de perseverança que sempre foram.

Aos meus irmãos Pedro e Rafael, que sempre me incentivaram e me apoiaram para que eu viesse a São Carlos cursar a faculdade de Matemática que eu tanto desejava. Sempre acreditaram na minha capacidade, mais do que eu mesma, me impulsionando a batalhar para alcançar meus objetivos.

Ao meu namorado Raphael, que esteve ao meu lado durante toda essa caminhada. Que por tantas vezes me ajudou a estudar para as provas, mesmo sem muito conhecimento daquelas matérias. Que foi tolerante e compreensivo. Seu amor e sua presença amenizaram imensamente as dificuldades dessa jornada.

Aos colegas de faculdade e de mestrado, em especial: Rodolfo, Luan, Henrique, Camilo, Steve e Dione, pelo companheirismo, paciência, por aquela ajuda tão importante ao longo dos semestres, e também pelos jogos de buraco, sempre divertidíssimos.

Às amigas que tive o prazer de conviver durante a faculdade: Martha, Rafaela, Michelle, Isaura e Daniela por todos esses anos de convivênvia em São Carlos. As festas, os passeios, as longas conversas e os jantares serão sempre lembrados com carinho e com a esperança de que essa amizade nunca acabe. 
A todos os professores que tive a oportunidade de conhecer ao longo dessa caminhada, que muito colaboraram para meu crescimento, em especial às minhas orientadoras de iniciação científica e mestrado Sandra e Sueli, respectivamente, sempre tão compreensivas, dedicadas e atenciosas e também à professora Iris, por me ajudar em diversas ocasiões.

Agradeço enfim ao Instituto de Ciências Matemáticas e de Computação, pela infraestrutura de qualidade oferecida aos alunos e por propiciar um ambiente favorável à construção do conhecimento e tambem ao CNPQ, pelo apoio financeiro. 


\section{Resumo}

Esta dissertação tem como objetivo principal estudar a estabilidade assintótica de Equações Diferenciais com Retardamento utilizando as técnicas desenvolvidas por Faria e Liz (2003). Para analisar a estabilidade assintótica, a abordagem utilizada neste trabalho é a de impor uma condição de dominância do termo sem retardo sobre a parte com retardo, possibilitando o estudo de soluções oscilatórias. Além disso, o estudo culminará em um teorema que garante essa estabilidade em uma Equação do tipo Lotka-Volterra, para a qual o método de Liapunov não se aplica. Para atingir o objetivo, estudou-se inicialmente a teoria geral de estabilidade de Equações Diferenciais Funcionais e a teoria de estabilidade para alguns tipos dessas equações. 


\section{Abstract}

This paper's main objective is to study the asymptotic stability of Differential Equations with Delay using the techniques developed by Faria and Liz (2003). To analyze the asymptotic stability, the approach used in this study is to impose a condition of dominance of the term without delay on the part delayed, allowing the study of oscillatory solutions. In addition, the study will culminate in a theorem ensures such stability in an equation Lotka-Volterra type, for which the Liapunov method does not apply. To achieve the objective, we studied initially the general theory of stability of Functional Differential Equations and stability theory for some types of these equations. 


\section{Introdução}

As Equações Diferenciais com Retardamento têm sido bastante utilizadas para modelar muitos problemas em Biologia e outras ciências, principalmente modelos de dinâmica populacional.

Existem muitos trabalhos que abordam as Equações do tipo Lotka-Volterra e Logísticas com Retardamento. Condições de limitação com relação ao número de coeficientes e de retardos são utilizadas para impor que soluções oscilatórias tendam a zero quando t tende a infinito.

Outra forma de se analisar a estabilidade assintótica é impor uma condição de dominância do termo sem retardo sobre a parte com retardo, possibilitando o estudo de soluções oscilatórias. É nesse contexto que este trabalho se encaixa. O objetivo é estudar as técnicas utilizadas por Faria e Liz (2003), no que diz respeito à estabilidade de Equações Diferenciais com Retardamento. Para isso, foi necessário estudar inicialmente a teoria geral de Equações Diferenciais com Retardamento e a Teoria de Estabilidade para alguns tipos dessas equações. O principal material utilizado foram os capítulos 1,2 e 5 de Hale e Lunel (1993).

O estudo da teoria básica e teoria de estabilidade das Equações Diferenciais Retardadas está descrito no Capítulo 1. Nele são apresentados conceitos relativos às equações diferenciais funcionais com retardo e notações que serão utilizados em toda a dissertação. Além disso, são abordados teoremas de existência, dependência contínua e unicidade das equações diferenciais funcionais retardadas.

O Capítulo 2 tem como objetivo investigar a estabilidade assintótica das Equações 
Logísticas com Retardo que são da forma:

$$
\dot{x}(t)=b(t) x(t)\left[1-L\left(x_{t}\right)\right]
$$

onde $b: \mathbb{R} \rightarrow \mathbb{R}$ é uma função contínua e $L: C([-r, 0] ; \mathbb{R}) \rightarrow \mathbb{R}$ é um operador linear positivo. São estabelecidas condições suficientes para garantir que soluções $x(t)$ oscilatórias ou não em torno de $x_{*}=\|L\|^{-1}$ tendam a $x_{*}$ quando $t$ tender ao infinito. Além disso, quando o operador $L$ tiver uma componente sem retardo que domine a parte com retardo, então o equilíbrio positivo $x_{*}$ será a única solução que é limitada e limitada inferiormente longe do zero.

Estendendo as técnicas empregadas no segundo capítulo a equações um pouco mais gerais, da forma:

$$
\dot{x}(t)=b(t) x(t)\left[a(t)-L\left(t, x_{t}\right)\right]
$$

cuja linearidade é não autônoma e $a: \mathbb{R} \rightarrow \mathbb{T}$ é uma função contínua, o Capítulo 3 traz condições que garantem que todas as soluções positivas dessa equação tendem ao equilíbrio positivo $x *$.

Já o Capítulo 4 destina-se ao estudo da Equação Logística Retardada Autônoma de forma mais geral, ou seja, diferentemente da equação vista no segundo capítulo, agora o operador $L$ não precisa ser positivo. Ainda assim, é possível garantir a estabilidade assintótica global do equilíbrio positivo, quando ele existir. Além disso, o estudo culminará em um teorema que garante essa estabilidade em uma Equação do tipo Lotka-Volterra, para a qual o método de Liapunov não se aplica. 


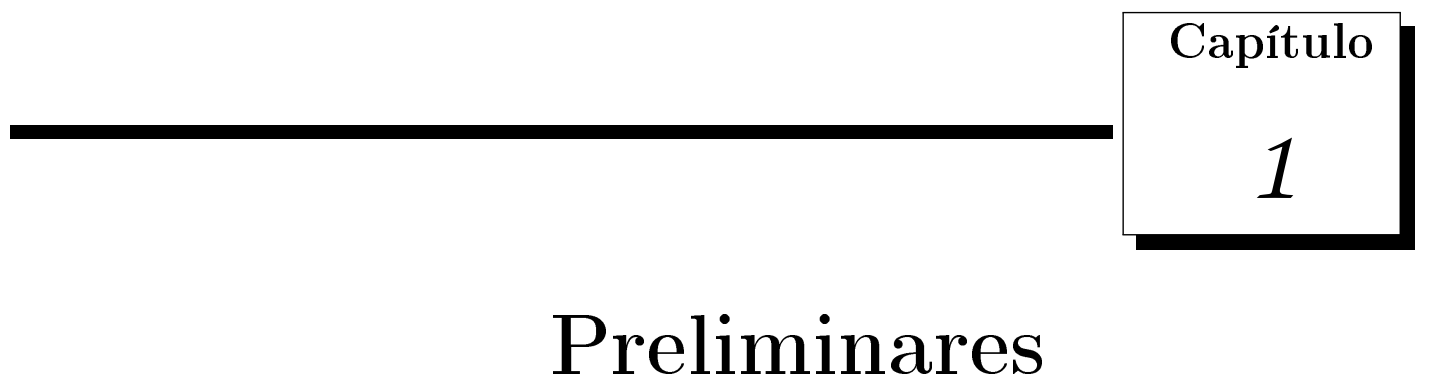

Neste capítulo, são apresentados alguns conceitos básicos, notações e resultados importantes que serão utilizados ao longo de toda a dissertação. Além disso, são abordados teoremas centrais relacionados à existência, unicidade e dependência contínua envolvendo as equações diferenciais funcionais com retardo.

\subsection{Alguns resultados importantes}

Os teoremas enunciados a seguir são bastante utilizados em diversas áreas da Matemática e desempenham um importante papel neste trabalho, auxiliando na demonstração de diversos resultados.

Teorema do Gráfico Fechado. Sejam E e F espaços de Banach e $T: E \rightarrow F$ um operador linear. Se $G_{T}=\{(x, y) \in E \times F ; y=T(x)\}$ é fechado em $E \times F$, então $T$ é contínuo.

Teorema da Convergência Dominada. Seja $f_{n}$ uma sequência de funções mensuráveis tal que $\left|f_{n}(x)\right| \leq g(x)$, com g integrável e $f_{n} \rightarrow f$ quase sempre. Então, $f$ é integrável $e$

$$
\lim \int f_{n} d \mu=\int f d \mu
$$


Teorema de Ascoli. Seja $W$ um espaço compacto. $K \subset C\left(W, \mathbb{R}^{n}\right)$ é relativamente compacto se, e somente se, $K$ é equicontinuo e uniformemente limitado.

Teorema do Ponto Fixo de Schauder. Se U é um subconjunto fechado, limitado e convexo de um espaço de Banach $X$ e $T: U \rightarrow U$ é completamente contínuo, então $T$ tem um ponto fixo em $U$.

\subsection{Equação Diferencial Funcional com Retardo - Con- ceitos básicos}

Definição 1.1. Defina $C=C([-r, 0] ; \mathbb{R})$ como sendo o espaço das funções contínuas de $[-r, 0]$ em $\mathbb{R}, r>0$, munido da norma:

$$
|\varphi|_{C}=\max _{-r \leq \theta \leq 0}|\varphi(\theta)|
$$

Definição 1.2. Considere $D$ um subconjunto de $\mathbb{R} \times C, f: D \rightarrow \mathbb{R}^{n}$ uma função e $x_{t} \in C$ definido por $x_{t}(\theta)=x(t+\theta)$, para $-r \leq \theta \leq 0$. A relação

$$
\dot{x}(t)=f\left(t, x_{t}\right)
$$

é uma Equação Diferencial Funcional com Retardo em D e pode ser denotada por EDFR ou, ainda, EDFR(f), quando se deseja enfatizar que tal equação é definida por $f$.

A seguir, alguns exemplos desse tipo de equação.

(i) Equação diferencial ordinária $(r=0): \dot{x}(t)=F(x(t))$.

(ii) Equação diferencial de diferença: $\dot{x}(t)=f\left(t, x(t), x\left(t-\tau_{1}(t)\right), \ldots, x\left(t-\tau_{p}(t)\right)\right)$, com $0 \leq \tau_{j}(t) \leq r, j=1,2, \ldots, p$.

(iii) Equação diferencial-integral: $\dot{x}(t)=\int_{-r}^{0} g(t, \theta, x(t+\theta)) d \theta$.

Definição 1.3. Uma função $x$ é uma solução de (1.1) em $[\sigma-r, \sigma+A)$ se existem $\sigma \in \mathbb{R} e$ $A>0$ tais que $x \in C\left([\sigma-r, \sigma+A), \mathbb{R}^{n}\right),\left(t, x_{t}\right) \in D$ e $x(t)$ satisfaz (1.1) para $t \in[\sigma, \sigma+A)$. 
Definição 1.4. Dados $\sigma \in \mathbb{R} e \phi \in C, x(\sigma, \phi, f)$ é uma solução da equação (1.1) com condição inicial $\phi$ em $\sigma$ se existe um $A>0$ tal que $x(\sigma, \phi, f)$ é uma solução de (1.1) em $[\sigma-r, \sigma+A)$ e $x_{\sigma}(\sigma, \phi, f)=\phi$. É equivalente dizer que $x(\sigma, \phi, f)$ é uma solução passando $\operatorname{por}(\sigma, \phi)$.

Definição 1.5. Uma solução $x(t)$ será limitada inferiormente longe do zero em um intervalo I quando existir uma constante positiva c tal que $x(t) \geq$ c para todo $t$ no intervalo I. Se existir uma sequência $\left(t_{n}\right) \subset \mathbb{R}$ satisfazendo $t_{n} \rightarrow \infty$ quando $n \rightarrow \infty$ e $x\left(t_{n}\right)=x_{*}$, para todo $n \in \mathbb{N}$, então $x(t)$ será uma solução oscilatória em torno de $x_{*}$. Caso contrário, ela será não oscilatória em torno de $x_{*}$.

Definição 1.6. A equação (1.1) é chamada de linear se $f(t, \phi)=L(t) \phi+h(t)$, com $L$ um operador linear. É autônoma se $f(t, \phi)=g(\phi)$, com g não dependendo de $t$.

Definição 1.7. Sendo $V$ um subconjunto de $\mathbb{R} \times C, C\left(V, \mathbb{R}^{n}\right)$ é a classe de todas as funções $f: V \rightarrow \mathbb{R}^{n}$ que são contínuas. Além disso, $C^{0}\left(V, \mathbb{R}^{n}\right) \subseteq C\left(V, \mathbb{R}^{n}\right)$ é o subconjunto das funções contínuas e limitadas de $V$ em $\mathbb{R}^{n}$ que, munido da norma

$$
|f|_{V}=\sup _{(t, \phi) \in V}|f(t, \phi)|
$$

torna-se um espaço de Banach.

Ao longo deste capítulo, as seguintes notações serão utilizadas:

Definição 1.8. Para qualquer $(\sigma, \phi) \in \mathbb{R} \times C$, considere $\widetilde{\phi} \in C\left([\sigma-r, \infty), \mathbb{R}^{n}\right)$ definido por

$$
\widetilde{\phi}_{\sigma}=\phi \quad e \quad \widetilde{\phi}(t+\sigma)=\phi(0), \quad t \geq 0
$$

Definição 1.9. Para quaisquer $\alpha$ e $\beta$ reais, define-se:

$$
\begin{array}{r}
I_{\alpha}=[0, \alpha], \quad B_{\beta}=\{\psi \in C:|\psi| \leq \beta\} \\
\mathcal{A}(\alpha, \beta)=\left\{y \in C\left([-r, \alpha], \mathbb{R}^{n}\right): y_{0}=0, y_{t} \in B_{\beta}, t \in I_{\alpha}\right\}
\end{array}
$$




\subsection{Resultados sobre existência, unicidade e dependên- cia contínua}

Os lemas a seguir são de grande importância para facilitar o entendimento e a demonstração dos teoremas centrais estudados neste capítulo.

Lema 1.1. Considere $\sigma \in \mathbb{R}, \phi \in C$ e $f(t, \phi)$ função continua. Encontrar uma solução da equação (1.1) passando por $(\sigma, \phi)$ é equivalente a resolver a equação integral

$$
\begin{gathered}
x_{\sigma}=\phi, \\
x(t)=\phi(0)+\int_{0}^{t} f\left(s, x_{s}\right) d s, \quad t \geq \sigma .
\end{gathered}
$$

Lema 1.2. Se $x \in C\left([\sigma-r, \sigma+\alpha], \mathbb{R}^{n}\right)$, então a função que associa $t$ à $x_{t}$ é contínua para $t \in[\sigma, \sigma+\alpha]$.

Prova: A função $x$ é contínua no intervalo fechado $[\sigma-r, \sigma+\alpha]$, que é compacto. Sendo assim, $x$ é uniformemente contínua. Logo, para $\varepsilon>0$ dado existe um $\delta>0$ tal que $|x(t)-x(\tau)|<\frac{\varepsilon}{2}$ quando $|t-\tau|<\delta$, quaisquer que sejam $t, \tau \in[\sigma-r, \sigma+\alpha]$. Consequentemente, para $t \in[\sigma, \sigma+\alpha],|t-\tau|<\delta$, segue que:

$$
|x(t+\theta)-x(\tau+\theta)|<\varepsilon \quad \text { para todo } \theta \in[-r, 0] .
$$

Portanto,

$$
\left|x_{t}-x_{\tau}\right|=\sup _{-r \leq \theta \leq 0}|x(t+\theta)-x(\tau+\theta)| \leq \frac{\varepsilon}{2}<\varepsilon
$$

e o resultado está demonstrado.

Lema 1.3. Considere $\Omega \subseteq \mathbb{R} \times C$ um aberto,$W \subseteq \Omega$ um compacto e $f^{0} \in C\left(\Omega, \mathbb{R}^{n}\right)$ uma função dada, então:

A) Existe uma vizinhança $V \subseteq \Omega$ de $W$ tal que $f^{0} \in C^{0}\left(V, \mathbb{R}^{n}\right)$. 
B) Existem uma vizinhança $U \subseteq C^{0}\left(V, \mathbb{R}^{n}\right)$ de $f^{0}$ e constantes positivas $M$, $\alpha$, $\beta$ tais que

$$
|f(\sigma, \phi)|<M \quad \text { para } \quad(\sigma, \phi) \in V \quad \text { e } \quad f \in U .
$$

C) Além disso, para qualquer $\left(\sigma^{0}, \phi^{0}\right) \in W,\left(\sigma^{0}+t, y_{t}+\widetilde{\phi}_{\sigma^{0}+t^{0}}\right) \in V$ para $t \in I_{\alpha} e$ $y \in \mathcal{A}(\alpha, \beta)$.

Prova: Como W é compacto e $f^{0}$ é contínua, existe uma constante $M>0$ tal que

$$
\left|f^{0}\left(\sigma^{0}, \phi^{0}\right)\right|<M, \quad \text { para } \quad\left(\sigma^{0}, \phi^{0}\right) \in W
$$

Além disso, é possível encontrar constantes positivas $\bar{\alpha}, \bar{\beta}$ e $\varepsilon$ tais que

$$
\left|f^{0}\left(\sigma^{0}+t, \phi^{0}+\psi\right)\right|<M-\varepsilon, \quad \forall\left(\sigma^{0}, \phi^{0}\right) \in W \quad e \quad(t, \psi) \in I_{\bar{\alpha}} \times B_{\bar{\beta}} .
$$

Suponha que não existam tais contantes. Então, para quaisquer $\bar{\alpha}, \bar{\beta}$ e $\varepsilon$ positivos existem $\left(\sigma^{0}, \phi^{0}\right) \in W$ e $(t, \psi) \in I_{\bar{\alpha}} \times B_{\bar{\beta}}$ tais que

$$
\left|f^{0}\left(\sigma^{0}+t, \phi^{0}+\psi\right)\right| \geq M-\varepsilon
$$

Considere $\overline{\alpha_{n}}, \overline{\beta_{n}}$ e $\varepsilon_{n}$ sequências convergindo para zero. Então existem $\left(\sigma_{n}^{0}, \phi_{n}^{0}\right) \in W$ e $\left(t_{n}, \psi_{n}\right) \in I_{\bar{\alpha}_{n}} \times B_{\bar{\beta}_{n}}$ tais que

$$
\left|f^{0}\left(\sigma_{n}^{0}+t_{n}, \phi_{n}^{0}+\psi_{n}\right)\right| \geq M-\varepsilon_{n} .
$$

Como W é compacto, $\sigma_{n}^{0}$ e $\phi_{n}^{0}$ possuem subsequências, aqui representadas com a mesma notação, que são convergentes, isto é, existe $\left(\sigma^{0}, \phi^{0}\right) \in W$ tais que $\sigma_{n}^{0} \rightarrow \sigma^{0}$ e $\phi_{n}^{0} \rightarrow \phi^{0}$. De acordo com (1.5) segue que

$$
\lim _{n \rightarrow \infty}\left|f^{0}\left(\sigma_{n}^{0}+t_{n}, \phi_{n}^{0}+\psi_{n}\right)\right| \geq \lim _{n \rightarrow \infty}\left(M-\varepsilon_{n}\right)
$$


e, como $f^{0}$ é contínua,

$$
\left|\lim _{n \rightarrow \infty} f^{0}\left(\sigma_{n}^{0}+t_{n}, \phi_{n}^{0}+\psi_{n}\right)\right| \geq M
$$

Portanto,

$$
\left|f^{0}\left(\sigma^{0}+\lim _{n \rightarrow \infty} t_{n}, \phi^{0}+\lim _{n \rightarrow \infty} \psi_{n}\right)\right| \geq M
$$

Por construção, $\bar{\alpha}_{n} \rightarrow 0$ e $t_{n} \in I_{\bar{\alpha}_{n}}=\left[0, \alpha_{n}\right]$, logo, $t_{n} \rightarrow 0$. Além disso, $\bar{\beta}_{n} \rightarrow 0$ e $\psi_{n} \in B_{\overline{\beta_{n}}}$, logo, $\left|\psi_{n}\right| \leq \bar{\beta}_{n}$, e conclui-se que $\psi_{n} \rightarrow 0$. Dessa forma, de (1.7) segue que

$$
\left|f^{0}\left(\sigma^{0}, \phi^{0}\right)\right| \geq M
$$

o que é uma contradição, pois, de acordo com (1.5), $\left|f^{0}\left(\sigma^{0}, \phi^{0}\right)\right|<M$ para todo $\left(\sigma^{0}, \phi^{0}\right) \in$ $W$.

Para concluir a prova do item A), basta definir

$$
V=\left\{\left(\sigma^{0}+t, \phi^{0}+\psi\right):\left(\sigma^{0}, \phi^{0}\right) \in W,(t, \psi) \in I_{\bar{\alpha}} \times B_{\bar{\beta}}\right\}
$$

e observar que $V \subseteq \Omega$ e $f^{0} \in C^{0}\left(V, \mathbb{R}^{n}\right)$.

B) Considere $\varepsilon$ obtido no item A) e defina

$$
U=\left\{f \in C\left(V, \mathbb{R}^{n}\right) ;\left|f-f^{0}\right|<\varepsilon\right\} .
$$

Sendo assim, se $(\sigma, \phi) \in V$ e $f \in U$, segue que

$$
|f(\sigma, \phi)| \leq\left|f(\sigma, \phi)-f^{0}(\sigma, \phi)\right|+\left|f^{0}(\sigma, \phi)\right|<\varepsilon+M-\varepsilon=M
$$

Portanto, $f \in C^{0}\left(V, \mathbb{R}^{n}\right)$, ou seja, $U \subseteq C^{0}\left(V, \mathbb{R}^{n}\right)$ é vizinhança de $f^{0}$ e $|f(\sigma, \phi)|<M$, para todo $(\sigma, \phi) \in V$ e $f \in U$.

C) Considere $\beta$ de tal modo que

$$
0<\beta<\bar{\beta}
$$


Dessa forma, pode-se tomar $\alpha$ de tal modo que

$$
\alpha<\bar{\alpha} \quad e \quad\left|\widetilde{\phi}_{\sigma^{0}+t^{0}}-\phi^{0}\right|<\bar{\beta}-\beta, \quad \forall\left(\sigma^{0}, \phi^{0}\right) \in W, \quad t \in I_{\alpha} .
$$

Suponha que não seja possível. Então, para cada $\alpha<\bar{\alpha}$ existem $\left(\sigma_{\alpha}^{0}, \phi_{\alpha}^{0}\right) \in W$ e $t_{\alpha} \in I_{\alpha}$ tais que $\left|\widetilde{\phi}_{\sigma_{\alpha}^{0}+t_{\alpha}}-\phi_{\alpha}^{0}\right| \geq \bar{\beta}-\beta$.

Considere $\alpha_{n}$ uma sequência convergindo pra zero. Como $t_{\alpha_{n}} \in\left[0, \alpha_{n}\right]$ para todo n, a sequência $t_{\alpha_{n}}$ também converge para zero. Além disso, $\left(\phi_{\alpha_{n}}^{0}, \phi_{\alpha_{n}}^{0}\right) \in W$ e

$$
\left|\widetilde{\sigma}_{\alpha_{n}^{0}+t_{\alpha_{n}}}-\phi_{\alpha_{n}}^{0}\right| \geq \bar{\beta}-\beta
$$

Sendo W compacto, as sequências $\sigma_{\alpha_{n}}^{0}$ e $\phi_{\alpha_{n}}^{0}$ possuem subsequências convergentes, aqui representadas com a mesma notação. Ou seja, existe $\left(\sigma^{0}, \phi^{0}\right) \in W$ tal que $\sigma_{\alpha_{n}}^{0} \rightarrow \sigma^{0}$ e $\phi_{\alpha_{n}}^{0} \rightarrow \phi^{0}$. Logo, por (1.9) segue que

$$
\left|\lim \widetilde{\phi}_{\sigma_{\alpha_{n}}^{0}+t_{\alpha_{n}}}-\lim \phi_{\alpha_{n}}^{0}\right| \geq \bar{\beta}-\beta
$$

Dessa forma,

$$
0=\left|\widetilde{\phi}_{\sigma^{0}}-\phi^{0}\right| \geq \bar{\beta}-\beta
$$

o que implica que $\bar{\beta} \leq \beta$, contradizendo (1.8) e, portanto, provando a existência de tal $\alpha$.

Considere $t \in I_{\alpha}, y \in \mathcal{A}(\alpha, \beta)$ e $\alpha$ e $\beta$ como descritos acima. Então, para todo $\left(\sigma^{0}, \phi^{0}\right) \in W$, conclui-se que $\left(\sigma^{0}+t, y_{t}+\widetilde{\phi}_{\sigma^{0}+t^{0}}\right) \in V$. Para isso, basta ver que $t \in I_{\widetilde{\alpha}}$ e que $y_{t}+\widetilde{\phi}_{\sigma^{0}+t^{0}}=\phi^{0}+y_{t}+\widetilde{\phi}_{\sigma^{0}+t^{0}}-\phi^{0}=\phi^{0}+\psi$, com $\psi \in B_{\bar{\beta}}$, pois, $\left|y_{t}+\widetilde{\phi}_{\sigma^{0}+t^{0}}-\phi^{0}\right| \leq$ $\left|y_{t}\right|+\left|\widetilde{\phi}_{\sigma^{0}+t^{0}}-\phi^{0}\right|<\beta+\bar{\beta}-\beta=\bar{\beta}$.

Lema 1.4. Considere $\Omega \in \mathbb{R} \times C$ um aberto, $W \subseteq \Omega$ um compacto e $f^{0} \in C\left(\Omega, \mathbb{R}^{n}\right)$ uma função dada. Considere também as vizinhanças $U, V$ e as constantes $M, \alpha$ e $\beta$ obtidas no Lema 1.3. Se

$$
T: W \times U \times \mathcal{A}(\alpha, \beta) \rightarrow C\left([-r, \alpha], \mathbb{R}^{n}\right)
$$


é definido por

$$
\begin{aligned}
& T(\sigma, \phi, f, y)(t)=0, \quad t \in[-r, 0] \\
& T(\sigma, \phi, f, y)(t)=\int_{0}^{t} f\left(\sigma+s, \widetilde{\phi}_{\sigma+s}+y_{s}\right) d s, \quad t \in[0, \alpha],
\end{aligned}
$$

então:

(A) Té contínuo.

(B) Existe um compacto $K$ em $C\left([-r, \alpha], \mathbb{R}^{n}\right)$ tal que

$$
T: W \times U \times \mathcal{A}(\alpha, \beta) \rightarrow K
$$

(C) Além disso, se $M \alpha \leq \beta$ então

$$
T: W \times U \times \mathcal{A}(\alpha, \beta) \rightarrow \mathcal{A}(\alpha, \beta) .
$$

Prova: Observe inicialmente que $T: W \times U \times \mathcal{A}(\alpha, \beta) \rightarrow C\left([-r, \alpha], \mathbb{R}^{n}\right)$.

B) O Lema 1.3 afirma que existem vizinhanças $V \subset \Omega$ de $\mathrm{W}$ tal que $f^{0} \in C^{0}\left(V, \mathbb{R}^{n}\right)$, $U \subset C^{0}\left(V, \mathbb{R}^{n}\right)$ de $f^{0}$ e constantes positivas $M, \alpha$ e $\beta$ tais que $|f(\sigma . \phi)<M|$, para todo $(\sigma, \phi) \in V$ e $f \in U$.

Além disso, para $t, \tau \in[-r, \alpha]$, segue que:

$$
|T(\sigma, \phi, f, y)(t)-T(\sigma, \phi, f, y)(\tau)|=0 \leq M|t-\tau|, \quad t, \tau \in[-r, 0] .
$$




$$
\begin{aligned}
|T(\sigma, \phi, f, y)(t)-T(\sigma, \phi, f, y)(\tau)| & =\left|\int_{0}^{t} f\left(\sigma+s, \widetilde{\phi}_{\sigma+s}+y_{s}\right) d s\right| \leq \int_{0}^{t}\left|f\left(\sigma+s, \widetilde{\phi}_{\sigma+s}+y_{s}\right)\right| d s \\
& \leq M t \leq M|t-\tau|, t \in I_{\alpha} e \tau \in[-r, 0] . \\
|T(\sigma, \phi, f, y)(t)-T(\sigma, \phi, f, y)(\tau)| & =\left|\int_{0}^{\tau} f\left(\sigma+s, \widetilde{\phi}_{\sigma+s}+y_{s}\right) d s\right| \leq \int_{0}^{\tau}\left|f\left(\sigma+s, \widetilde{\phi}_{\sigma+s}+y_{s}\right)\right| d s \\
& \leq M \tau \leq M|t-\tau|, t \in[-r, 0] e \tau \in I_{\alpha} . \\
|T(\sigma, \phi, f, y)(t)-T(\sigma, \phi, f, y)(\tau)| & =\left|\int_{t}^{\tau} f\left(\sigma+s, \widetilde{\phi}_{\sigma+s}+y_{s}\right) d s\right| \leq \int_{t}^{\tau}\left|f\left(\sigma+s, \widetilde{\phi}_{\sigma+s}+y_{s}\right)\right| d s \\
& \leq M|t-\tau|, t, \tau \in I_{\alpha} .
\end{aligned}
$$

Dessa forma, conclui-se que, para $t, \tau \in[-r, \alpha]$,

$$
|T(\sigma, \phi, f, y)(t)-T(\sigma, \phi, f, y)(\tau)| \leq M|t-\tau|
$$

Defina

$$
K=\left\{g \in C\left([-r, \alpha], \mathbb{R}^{n}\right) ;|g(t)-g(\tau)| \leq M|t-\tau| e|g(t)| \leq M \alpha\right\}
$$

Para completar a prova do item B), resta mostrar que K é compacto. Para isso, é suficiente provar que $\bar{K}$ é compacto e $\mathrm{K}$ é fechado.

Para $g \in K$, tem-se:

$$
\|g\|=\sup _{t \in[-r, \alpha]}|g(t)| \leq M \alpha
$$

Logo,

$$
|g(t)| \leq M \alpha, \forall t \in[-r, \alpha], \forall g \in K
$$

Tome $\varepsilon>0$ arbitrário e considere $\delta=\frac{\varepsilon}{M}$. Se $t, \tau \in[-r, \alpha]$ são tais que $|t-\tau|<\delta$, segue que $|g(t)-g(\tau)| \leq M|t-\tau|<M \delta<\varepsilon$, para todo $g \in K$.

Portanto, $\bar{K}$ é uniformemente limitado e equicontínuo e o Teorema de Ascoli garante que $\bar{K}$ é compacto.

Considere agora uma sequência $\left(g_{n}\right) \subset K$ que convirja para $g$. Dessa forma, para cada $\mathrm{n}, g_{n} \in C\left([-r, \alpha], \mathbb{R}^{n}\right)$, logo, $g \in C\left([-r, \alpha], \mathbb{R}^{n}\right)$. Além disso, $\left|g_{n}(t)-g_{n}(\tau)\right| \leq M|t-\tau|$. 
Logo, $\lim \left|g_{n}(t)-g_{n}(\tau)\right| \leq M|t-\tau|$, o que implica que $|g(t)-g(\tau)| \leq M|t-\tau|$.

E como $\left|g_{n}(t)\right| \leq M \alpha$ para todo $n$, segue que $|g(t)| \leq M \alpha$. Portanto $g \in K$ e, consequentemente, $\mathrm{K}$ é fechado, completando a prova.

A) De acordo com o Teorema do Gráfico Fechado, é suficiente provar que T é fechado para garantir que T seja contínuo.

Dessa forma, considere $\left(\sigma^{k}, \phi^{k}, f^{k}, y^{k}\right),\left(\sigma^{0}, \phi^{0}, f^{0}, y^{0}\right)$ sequências em $W \times U \times \mathcal{A}(\alpha, \beta)$ tais que $\left(\sigma^{k}, \phi^{k}, f^{k}, y^{k}\right) \rightarrow\left(\sigma^{0}, \phi^{0}, f^{0}, y^{0}\right)$.

Observe que $T\left(\sigma^{k}, \phi^{k}, f^{k}, y^{k}\right) \in K$, que é compacto. Logo, existe uma subsequência de $T\left(\sigma^{k}, \phi^{k}, f^{k}, y^{k}\right)$, aqui representada com a mesma notação, e $\gamma \in K$, de tal modo que

$$
T\left(\sigma^{k}, \phi^{k}, f^{k}, y^{k}\right) \rightarrow \gamma
$$

Para $t$ no intervalo $[-r, 0]$, vale: $T\left(\sigma^{k}, \phi^{k}, f^{k}, y^{k}\right)(t)=T\left(\sigma^{0}, \phi^{0}, f^{0}, y^{0}\right)(t)=0$. Logo, $\lim _{k \rightarrow \infty} T\left(\sigma^{k}, \phi^{k}, f^{k}, y^{k}\right)(t)=0$. E, portanto, $\gamma(t)=T\left(\sigma^{0}, \phi^{0}, f^{0}, y^{0}\right)(t)$.

Quando $t \in I_{\alpha}, \lim _{k \rightarrow \infty} T\left(\sigma^{k}, \phi^{k}, f^{k}, y^{k}\right)(t)=\lim _{k \rightarrow \infty} \int_{0}^{t} f^{k}\left(\sigma^{k}+s, \widetilde{\phi}_{\sigma^{k}+s}+y_{s}\right) d s$.

De acordo com o Lema 1.3, todas essas $f^{k}$ são limitadas. Além disso, para todo $s \in I_{\alpha}$, $f^{k}\left(\sigma^{k}+s, \widetilde{\phi}_{\sigma^{k}+s}+y_{s}\right) \rightarrow f^{0}\left(\sigma^{0}+s, \widetilde{\phi}_{\sigma^{0}+s}+y_{s}\right)$. Dessa forma, pode-se aplicar o Teorema da Convergência Dominada e obtém-se que

$$
\int_{0}^{t} f^{k}\left(\sigma^{k}+s, \widetilde{\phi}_{\sigma^{k}+s}+y_{s}\right) d s \rightarrow \int_{0}^{t} f^{0}\left(\sigma^{0}+s, \widetilde{\phi}_{\sigma^{0}+s}+y_{s}\right)
$$

Logo,

$$
\gamma(t)=\int_{0}^{t} f^{0}\left(\sigma^{0}+s, \widetilde{\phi}_{\sigma^{0}+s}+y_{s}\right) d s=T\left(\sigma^{0}, \phi^{0}, f^{0}, y^{0}\right)(t)
$$

Portanto,

$$
T\left(\sigma^{k}, \phi^{k}, f^{k}, y^{k}\right) \rightarrow T\left(\sigma^{0}, \phi^{0}, f^{0}, y^{0}\right)
$$

Observe que esse resultado é válido para qualquer subsequência de $T\left(\sigma^{k}, \phi^{k}, f^{k}, y^{k}\right)$ que convirja. E, assim, o seguinte resultado é valido:

Proposição 1.1. Seja $\left(x_{n}\right)$ sequência contida em um compacto K. Se existir uma subsequência $\left(x_{n_{k}}\right)$ convergindo para $x$ e qualquer subsequência convergente de $\left(x_{n}\right)$ também convergir para $x$, então $x_{n}$ converge para $x$, 
permitindo concluir que $\mathrm{T}$ é fechado, consequentemente contínuo.

C) Suponha que $M \alpha \leq \beta$ e tome $T(\sigma, \phi, f, y) \in K$. Então, $T(\sigma, \phi, f, y) \in C\left(\left[-r, \alpha, \mathbb{R}^{n}\right]\right)$ e $T(\sigma, \phi, f, y)_{0}=T(\sigma, \phi, f, y)(\theta)$, com $\theta \in[-r, 0]$. Sendo assim, $T(\sigma, \phi, f, y)_{0}=0$ e, para $t \in I_{\alpha}$ e $\theta \in[-r, 0],\left\|T(\sigma, \phi, f, y)_{t}\right\|=|T(\sigma, \phi, f, y)(t+\theta)| \leq M \alpha \leq \beta$.

Portanto, $T(\sigma, \phi, f, y) \in \mathcal{A}(\alpha, \beta)$, ou seja, $T: W \times U \times \mathcal{A}(\alpha, \beta) \rightarrow \mathcal{A}(\alpha, \beta)$.

Teorema 1.2. (Existência). Considere $\Omega$ um subconjunto aberto de $\mathbb{R} \times C$. Se $W \subseteq \Omega$ é compacto e $f^{0} \in C\left(\Omega, \mathbb{R}^{n}\right)$ uma função, então existem uma vizinhança $V \subseteq \Omega$ de $W$ tal que $f^{0} \in C^{0}\left(V, \mathbb{R}^{n}\right)$, uma vizinhança $U \subseteq C^{0}\left(V, \mathbb{R}^{n}\right)$ de $f^{0}$ e um $\alpha>0$ tal que, para qualquer $(\sigma, \phi) \in W$, existe uma solução $x\left(\sigma, \phi, f^{0}\right)$ da $\operatorname{RFDE}\left(f^{0}\right)$ em $[\sigma-r, \sigma+\alpha]$ passando por $(\sigma, \phi)$.

Prova: Considere $(\sigma, \phi) \in \Omega$ e tome as vizinhanças $\mathrm{U}, \mathrm{V}$ e as constantes $M, \alpha$ e $\beta$ obtidas no Lema $1.3 \operatorname{com} M \alpha \leq \beta$.

Afirmação 1: $\mathcal{A}(\alpha, \beta)$ é um subconjunto fechado, convexo e limitado de $C\left([-r, \alpha], \mathbb{R}^{n}\right)$.

Considere $\lambda \in[0,1]$ e $x, y \in \mathcal{A}(\alpha, \beta)$. Dessa forma, $x_{0}=0=y_{0}$ e $x_{t}, y_{t} \in B_{\beta}$ para todo $t \in I_{\alpha}$. Logo,

$$
\begin{aligned}
& (1-\lambda) x_{0}+\lambda y_{0}=0, \quad(1-\lambda) x+\lambda y \in C e \\
& \left|(1-\lambda) x_{t}+\lambda y_{t}\right| \leq|1-\lambda|\left|x_{t}\right|+|\lambda|\left|y_{t}\right| \leq|1-\lambda| \beta+\lambda \beta \leq \beta,
\end{aligned}
$$

o que mostra que $\mathcal{A}(\alpha, \beta)$ é convexo.

Provar que $\mathcal{A}(\alpha, \beta)$ é limitado é encontrar $R>0$ de tal forma que $\mathcal{A}(\alpha, \beta) \subset B(0, R)$. Para isso, tome $R=\beta$ e considere $y \in \mathcal{A}(\alpha, \beta)$. Então

$$
|y|=\sup _{-r \leq \theta \leq \alpha}|y(t)| \leq \beta
$$

pois, por pertencer à $\mathcal{A}(\alpha, \beta)$ segue que $y(t)=y_{0}=0$, para $t \in[-r, 0]$ e $y(t)=y_{t}$, para $t \in[0, \alpha]$. Além disso, $\left|y_{t}\right| \leq \beta$, já que $y_{t} \in B_{\beta}$, para todo $t \in[0, \alpha]$.

Portanto, $y \in B(0, R)$, para $R=\beta$, ou seja, $\mathcal{A}(\alpha, \beta)$ é limitado.

Falta mostrar que $\mathcal{A}(\alpha, \beta)$ é fechado. Para isso, considere uma sequência $\left(y_{n}\right) \subset$ 
$\mathcal{A}(\alpha, \beta)$ tal que $y_{n} \rightarrow y$. Assim,

$$
\begin{aligned}
|y|= & \sup _{t \in I_{\alpha}}\left|y_{t}\right|=\sup _{t \in I_{\alpha}}|y(t+\theta)|=\sup _{t \in I_{\alpha}}\left|\lim _{\theta \in[-r, 0]} y_{n \rightarrow \infty}(t+\theta)\right| \\
& \leq \limsup _{n \rightarrow \infty}\left|y_{n}(t+\theta)\right| \leq \lim _{n \rightarrow \infty} \beta \leq \beta,
\end{aligned}
$$

$\operatorname{logo}, y \in \mathcal{A}(\alpha, \beta)$, completando a prova da Afirmação 1 .

Afirmação 2: o operador

$$
\begin{aligned}
F: \mathcal{A}(\alpha, \beta) & \rightarrow \mathcal{A}(\alpha, \beta) \\
y & \mapsto F_{y}=T\left(\sigma, \phi, f^{0}, y\right),
\end{aligned}
$$

definido como em (1.10), é completamente contínuo.

Inicialmente, observe que $\mathcal{A}(\alpha, \beta) \subset C^{0}\left([-r, \alpha], \mathbb{R}^{n}\right)$ e que $C^{0}\left([-r, \alpha], \mathbb{R}^{n}\right)$ é um espaço de Banach com a norma descrita Definição 1.7. Observe também que F está bem definido. De fato, para $y \in \mathcal{A}(\alpha, \beta), F_{y} \in C\left([-r, \alpha], \mathbb{R}^{n}\right)$ e $F_{y_{0}}=F_{y}(0+\theta)=F_{y}(\theta)=0$, para $\theta \in[-r, 0]$. E, para $t \in I_{\alpha}$, segue que

$$
\begin{aligned}
\left|F_{y_{t}}\right|_{C} & =\sup _{-r \leq \theta \leq 0}\left|F_{y_{t}}(\theta)\right|=\sup _{-r \leq \theta \leq \alpha}\left|F_{y}(t+\theta)\right|=\sup _{-r \leq \theta \leq 0}\left|\int_{0}^{t+\theta} f^{0}\left(\sigma+s, \widetilde{\phi}_{\sigma+s}+y_{s}\right) d s\right| \\
& \leq \sup _{-r \leq \theta \leq \alpha} \int_{0}^{t+\theta}\left|f^{0}\left(\sigma+s, \widetilde{\phi}_{\sigma+s}+y_{s}\right)\right| d s \leq M \alpha \leq \beta .
\end{aligned}
$$

Além disso, F é um operador contínuo. Dessa forma, resta mostrar que, dado $L \subset$ $\mathcal{A}(\alpha, \beta)$ limitado, é verdade que $\overline{F(L)}=\overline{T\left(\sigma, \phi, f^{0}, L\right)}=A$ é compacto.

O Lema 1.4 afirma que existe $K \subset C\left([-r, \alpha], \mathbb{R}^{n}\right)$ compacto tal que $T: W \times U \times$ $\mathcal{A}(\alpha, \beta) \rightarrow K$. Logo, $T\left(\sigma, \phi, f^{0}, L\right) \subset K$ e, portanto, $\overline{T\left(\sigma, \phi, f^{0}, L\right)}=A \subset \bar{K}=K$. Dessa forma, A é limitado. Mais ainda, A é uniformemente limitado.

Além disso, A é equicontínuo. Para mostrar que esse fato é verdadeiro, considere $f \in A$. Então, existe uma sequência $\left(f_{n}\right) \subset T\left(\sigma, \phi, f^{0}, L\right)$ tal que $f_{n} \rightarrow f$. Isto é, dado $\varepsilon>0$, existe um $N$ de tal modo que, se $n \geq N$, segue que $\left|f_{n}-f\right| \leq \frac{\varepsilon}{4}$. Tome $\delta=\frac{\varepsilon}{2 M}$. 
Para $t, \tau$ satisfazendo $|t-\tau|<\delta$, tem-se que:

$$
\begin{aligned}
|f(t)-f(\tau)| & \leq\left|f(t)-f_{N}(t)\right|+\left|f_{N}(t)-f_{N}(\tau)\right|+\left|f_{N}(\tau)-f(\tau)\right| \\
& \leq \frac{\varepsilon}{4}+\left|T\left(\sigma, \phi, f^{0}, L_{N}\right)(t)-T\left(\sigma, \phi, f^{0}, L_{N}\right)(\tau)\right|+\frac{\varepsilon}{4} \\
& \leq \frac{\varepsilon}{2}+M|t-\tau|<\frac{\varepsilon}{2}+M \delta=\varepsilon .
\end{aligned}
$$

Portanto, A é uniformemente limitado e equicontínuo e, de acordo com o Teorema de Ascoli, $\bar{A}$ é compacto, ou seja, A é compacto.

Sendo verdadeiras as Afirmações 1 e 2, o Teorema do Ponto Fixo de Schauder garante que $\mathrm{F}$ tem um único ponto fixo, isto é, que existe $y \in \mathcal{A}(\alpha, \beta)$ tal que

$$
T\left(\sigma, \phi, f^{0}, y\right)=y
$$

ou seja,

$$
y_{0}=0 \quad e \quad y(t)=\int_{0}^{t} f^{0}\left(\sigma+s, \widetilde{\phi}_{\sigma+s}+y_{s}\right) d s, t \in I_{\alpha} .
$$

E o Lema 1.1 permite concluir que existe uma única solução passando por $(\sigma, \phi)$, o que prova o teorema.

Teorema 1.3. (Dependência continua). Considere $\Omega \subseteq \mathbb{R} \times C$ um aberto, $\left(\sigma^{0}, \phi^{0}\right) \in \Omega$, $f^{0} \in C\left(\Omega, \mathbb{R}^{n}\right)$ e $x^{0}$ uma solução da $\operatorname{EDFR}\left(f^{0}\right)$ passando por $\left(\sigma^{0}, \phi^{0}\right)$ que existe e é única em $\left[\sigma^{0}-r, b\right]$. Tome $W^{0} \subseteq \Omega$ compacto definido por

$$
W^{0}=\left\{\left(t, x_{t}^{0}\right): t \in\left[\sigma^{0}, b\right]\right\}
$$

e $V^{0}$ uma vizinhança de $W^{0}$ na qual $f^{0}$ é limitada.

Se $\left(\sigma^{k}, \phi^{k}, f^{k}\right), k=1,2, \ldots$ são sequências satisfazendo $\sigma^{k} \rightarrow \sigma^{0}, \phi^{k} \rightarrow \phi^{0}$ e $\mid f^{k}-$ $\left.f^{0}\right|_{V^{0}} \rightarrow 0$, quando $k \rightarrow \infty$, então existe um $k^{0}$ de tal forma que a $\operatorname{EDFR}\left(f^{k}\right)$, para $k \geq k^{0}$, é tal que cada solução $x^{k}=x^{k}\left(\sigma^{k}, \phi^{k}, f^{k}\right)$ passando por $\left(\sigma^{k}, \phi^{k}\right)$ existe em $\left[\sigma^{k}-r, b\right]$ e $x^{k} \rightarrow x^{0}$ uniformemente em $\left[\sigma^{0}-r, \beta\right]$.

Como todo $x^{k}$ pode não estar definido em $\left[\sigma^{0}-r, b\right]$ por $x^{k} \rightarrow x^{0}$ uniformemente em 
$\left[\sigma^{0}-r, b\right]$, isso quer dizer que, para qualquer $\epsilon>0$, existe um $k_{1}(\epsilon)$ tal que $x^{k}(t), k \geq k_{1}(\epsilon)$, está definida em $\left[\sigma^{0}-r+\epsilon, b\right]$ e $x^{k} \rightarrow x^{0}$ uniformemente em $\left[\sigma^{0}-r+\epsilon, b\right]$.

Prova: Considere sequências $\left(\sigma^{k}, \phi^{k}\right) \in \Omega$ satisfazendo $\sigma^{k} \rightarrow \sigma^{0}$ e $\phi^{k} \rightarrow \phi^{0}$.

Observe inicialmente que, sendo $W^{0}$ compacto, o conjunto $W^{0} \cup\left\{\left(\sigma^{k}, \phi^{k}\right)\right\}$ também é compacto. Para demonstrar esse fato, considere $\bigcup_{i=1}^{\infty} A_{i}$ cobertura aberta de $W^{0} \cup$ $\left\{\left(\sigma^{k}, \phi^{k}\right)\right\}$. Suponha, sem perda de generalidade que $\left(\sigma^{0}, \phi^{0}\right) \in A_{1}$.

Como $\left(\sigma^{k}, \phi^{k}\right) \rightarrow\left(\sigma^{0}, \phi^{0}\right)$, existem apenas finitos índices $k$ tais que $\left(\sigma^{k}, \phi^{k}\right) \notin A_{1}$. Sendo assim, é possível cobrir $\left\{\left(\sigma^{k}, \phi^{k}\right)\right\}$ com um número finito de $A_{i}$ 's. Além disso, por ser compacto, $W^{0}$ possui subcobertura finita. Portanto, $W^{0} \cup\left\{\left(\sigma^{k}, \phi^{k}\right)\right\}$ possui subcobertura finita; logo, é compacto.

Como $\left(\sigma^{k}, \phi^{k}\right) \rightarrow\left(\sigma^{0}, \phi^{0}\right) \in W^{0} \subset V^{0}$, existe $k_{1} \in \mathbb{N}$ tal que $\left(\sigma^{k}, \phi^{k}\right) \in V^{0}$, para todo $k \geq k_{1}$.

Defina o compacto

$$
W^{\prime}=W^{0} \cup\left\{\left(\sigma^{k}, \phi^{k}\right) ; k \geq k_{1}\right\} \subset V^{0}
$$

O Lema 1.3 afirma que existem $V \subset V^{0}$ vizinhança de $W^{\prime}$ tal que $f^{0} \in C^{0}\left(V, \mathbb{R}^{n}\right)$, $U \subset C^{0}\left(V, \mathbb{R}^{n}\right)$ vizinhança de $f^{0}$ e constantes $M, \alpha$ e $\beta>0$ tais que

$$
|f(\sigma, \phi)|<M, \forall(\sigma, \phi) \in V \text { e } f \in U
$$

Se $t \in I_{\alpha}$ e $y \in B_{\beta}$, então $\left(\sigma^{0}+t, \widetilde{\phi}_{\sigma^{0}+t}^{0}+y_{t}\right) \in V$ para todo $\left(\sigma^{0}, \phi^{0}\right) \in W^{\prime}$. Como $f^{k} \rightarrow f^{0}$ uniformemente em $V^{0}$ e $f^{0} \in U$, existe $k_{2} \in \mathbb{N}$ tal que $f^{k} \in U$, para todo $k \geq k_{2}$. Tome

$$
W=W^{0} \cup\left\{\left(\sigma^{k}, \phi^{k}\right), k \geq k_{0}\right\},
$$

onde $k_{0}=\max \left\{k_{1}, k_{2}\right\}$.

Para todo $k \geq k_{0}, f^{k} \in U$ e $\left(\sigma^{k}, \phi^{k}\right) \in W$ o Teorema 1.1 afirma que existe $\alpha_{0}>0$ tal que $x^{k}=x^{k}\left(\sigma^{k}, \phi^{k}, f^{k}\right)$ passa por $\left(\sigma^{k}, \phi^{k}\right)$ e está definida em $\left[\sigma^{k}-r, \sigma^{k}+\alpha_{0}\right], \alpha_{0}$ não dependendo de $\mathrm{k}$. 
Tome $y^{k}(t)=x^{k}\left(\sigma^{k}+t\right)-\widetilde{\phi}^{k}\left(\sigma^{k}+t\right)$, para todo $k \geq k_{0}$, onde $y^{k}$ é solução de

$$
\begin{gathered}
y_{0}=0 \\
y(t)=\int_{0}^{t} f^{k}\left(\sigma^{k}+s, \widetilde{\phi}_{\sigma^{k}+s}^{k}+y_{s}\right) d s, t \in I_{\alpha_{0}} .
\end{gathered}
$$

Pelo Lema 1.4, $y^{k}=T\left(\sigma^{k}, \phi^{k}, f^{k}, y^{k}\right) \subset C\left(\left[-r, \alpha_{0}\right], \mathbb{R}^{n}\right)$ e, portanto, existe uma subsequência $y^{k_{j}}$ de $y^{k}$ tal que $y^{k_{j}} \rightarrow y^{*}$ uniformemente em $\left[-r, \alpha_{0}\right]$, com $y^{*} \in C\left(\left[-r, \alpha_{0}\right], \mathbb{R}^{n}\right)$.

T é contínuo e $\left(\sigma^{k_{j}}, \phi^{k_{j}}, f^{k_{j}}, y^{k_{j}}\right) \rightarrow\left(\sigma^{0}, \phi^{0}, f^{0}, y^{0}\right)$. Logo, $y^{k_{j}}=T\left(\sigma^{k_{j}}, \phi^{k_{j}}, f^{k_{j}}, y^{k_{j}}\right) \rightarrow$ $T\left(\sigma^{0}, \phi^{0}, f^{0}, y^{0}\right)$. Portanto,

$$
T\left(\sigma^{0}, \phi^{0}, f^{0}, y^{0}\right)=y^{*}
$$

É verdade que $x^{0}$ é a única solução de $\dot{x}=f\left(t, x_{t}\right), x_{\sigma^{0}}=\phi^{0}$ e, portanto, $y^{0}(t)=$ $x^{0}\left(t+\sigma^{0}\right)-\widetilde{\phi}^{0}\left(\sigma^{0}+t\right), t \in\left[-r, \alpha_{0}\right]$ é a única solução de $y=T\left(\sigma^{0}, \phi^{0}, f^{0}, y^{0}\right)$. Logo (1.11) permite concluir que $y^{0}=y^{*}$.

Dessa maneira, $y^{k}$ converge para $y^{0}$ em $C\left(\left[-r, \alpha_{0}\right], \mathbb{R}^{n}\right)$.

Como para cada $k \geq k_{0}, x^{k}$ existe em $\left[\sigma^{k}-r, \sigma^{k}+\alpha_{0}\right]$ e $\sigma^{k} \rightarrow \sigma^{0}$, dado $\varepsilon>0$, existe $k_{1}^{\prime}=k_{1}^{\prime}\left(k_{1}, \varepsilon\right)$ tal que para todo $k \geq k_{1}^{\prime}, x^{k}$ existe em $\left[\sigma^{0}-r+\varepsilon, \sigma^{0}+\alpha_{0}-\varepsilon\right]$.

Como $y^{k} \rightarrow y^{0}$ em $C\left(\left[-r, \alpha_{0}\right], \mathbb{R}^{n}\right)$, a convergência é uniforme.

Logo, para $k \geq \max \left\{k_{0}, k_{1}^{\prime}\right\}$, segue que $x^{k}\left(\sigma^{k}+t\right)-\widetilde{\phi}^{k}\left(\sigma^{k}+t\right)=y^{k}(t) \rightarrow y^{0}(t)=$ $x^{0}\left(\sigma^{0}+t\right)-\widetilde{\phi}^{0}\left(\sigma^{0}+t\right)$ uniformemente, mas $\widetilde{\phi}^{k} \rightarrow \widetilde{\phi}^{0}$ uniformemente, pois, $\phi^{k} \rightarrow \phi^{0} \mathrm{em}$ $C\left([-r, 0], \mathbb{R}^{n}\right)$. Portanto, $x^{k}\left(\sigma^{k}+t\right) \rightarrow x^{0}$ uniformemente em $\left[\sigma^{0}-r+\varepsilon, \sigma^{0}+\alpha_{0}-\varepsilon\right]$.

Se $\sigma^{0}+\alpha_{0}>b$, o teorema está provado.

Se $\sigma^{0}+\alpha_{0} \leq b$; existe $k^{\prime} \geq 0$ tal que, para todo $k \geq k^{\prime}, \sigma^{k}+\alpha_{0} \leq b$.

Definindo $\sigma_{1}^{k}=\sigma^{k}+\alpha_{0}$ e $\phi_{1}^{k}=x_{\sigma_{1}^{k}}$, para todo $k \geq k^{\prime}$, obtém-se que $\sigma^{0}+\alpha_{0}=\phi_{1}^{0} \mathrm{e}$ $x_{\sigma^{0}+\alpha_{0}}=\phi_{1}^{0}$

Assim, é obtida uma sequência $\left(\alpha_{n}\right)$ de números positivos não nulos e, definindo

$$
\xi_{n}=\sum_{i=1}^{n} \alpha_{i}
$$

segue que $\left(\xi_{n}\right) \subset\left[\sigma^{0}-r, b\right],\left(\xi_{n}\right)$ é crescente e, portanto, existe $\bar{\alpha}=\sup _{n} \xi_{n}$.

Note que, se $\bar{\alpha}+\sigma^{0}<b$, existe $k_{1} \geq 0$ tal que $k \geq k_{1}$ implica que $\sigma^{k}+\bar{\alpha}<b$. 
Tomando $\bar{\sigma}^{k}=\sigma^{k}+\bar{\alpha}$ e $\bar{\phi}^{k}=x_{\sigma^{k}}+\bar{\alpha}$, aplica-se novamente a parte anterior e obtém-se $\alpha^{*}$ tal que, para $k \geq k^{*}, x^{k}$ é solução de $\dot{x}=f^{k}\left(t, x_{t}\right)$ passando por $\left(\bar{\sigma}^{k}, \bar{\phi}^{k}\right)$ que existe em $\left[\bar{\sigma}^{k}-\bar{r}, \bar{\sigma}^{k}+\alpha^{*}\right]$ e $x^{k} \rightarrow x^{0}$ uniformemente em $\left[\bar{\sigma}^{0}-\bar{r}, \bar{\sigma}^{0}+\alpha^{*}\right]$, o que contraria o fato de $\bar{\alpha}=\sup _{n} \sum_{i=1}^{n} \alpha_{i}$.

Desse modo, $\bar{\alpha}+\sigma^{0} \geq b$.

Teorema 1.4. (Unicidade) Considere $\Omega$ um aberto em $\mathbb{R} \times C, f: \Omega \rightarrow \mathbb{R}^{n}$ uma função contínua tal que $f(t, \phi)$ é Lipschitiziana em $\phi$ em cada compacto em $\Omega$. Se $(\sigma, \phi) \in \Omega$, então existe uma única solução de (1.1) passando por $(\sigma, \phi)$.

Prova: Suponha que $x$ e $y$ sejam soluções da equação (1.1) em $[\sigma-r, \sigma+\alpha]$. De acordo com o Lema 1.1, $x$ e $y$ satisfazem:

$$
\begin{array}{lll}
x_{\sigma}=\phi & e & x(t)=\phi(0)+\int_{\sigma}^{t} f\left(s, x_{s}\right) d s, \\
y_{\sigma}=\phi & e & y(t)=\phi(0)+\int_{\sigma}^{t} f\left(s, y_{s}\right) d s .
\end{array}
$$

Considere os compactos

$$
W_{x}=\left\{\left(t, x_{t}\right) ; t \in[\sigma, \sigma+\alpha]\right\} \quad e \quad W_{y}=\left\{\left(t, y_{t}\right) ; t \in[\sigma, \sigma+\alpha]\right\}
$$

Então, $W=W_{x} \times W_{y}$ também é compacto. Sendo assim, tome $k=k(W)$ a constante de Lipschtz de $f(t, \phi)$ em W e $\alpha_{1} \in[0, \alpha]$ satisfazendo $k \alpha_{1}=\lambda<1$.

Se $t \in\left[\sigma, \sigma+\alpha_{1}\right]$, então

$$
\begin{aligned}
|x(t)-y(t)| & =\left[\int_{\sigma}^{t}\left[f\left(s, x_{s}\right)-f\left(s, y_{s}\right)\right] d s\right] \leq \int_{\sigma}^{t}\left[f\left(s, x_{s}\right)-f\left(s, y_{s}\right)\right] d s \\
& \leq \int_{\sigma}^{t} k\left|x_{s}, y_{s}\right| d s \leq k|t-\sigma| \sup _{s \in[\sigma, t]}\left|x_{s}-y_{s}\right| \\
& \leq k \alpha_{1} \sup _{s \in[\sigma, t]}\left|x_{s}-y_{s}\right| \leq \lambda \sup _{s \in[\sigma, t]}\left|x_{s}-y_{s}\right|, \lambda \in[0,1) \\
& \leq \lambda \sup _{s \in\left[\sigma, \sigma+\alpha_{1}\right]}\left|x_{s}-y_{s}\right| \leq \lambda \sup _{t \in\left[\sigma-r, \sigma+\alpha_{1}\right]}|x(t)-y(t)| .
\end{aligned}
$$


E, portanto,

$$
\sup _{t \in\left[\sigma, \sigma+\alpha_{1}\right]}|x(t)-y(t)| \leq \lambda \sup _{t \in \sigma-r, \sigma+\alpha_{1}}|x(t)-y(t)| .
$$

Note que, se $t \in[\sigma-r, \sigma]$, então $x(t)-y(t)=x(\sigma+\theta)-y(\sigma+\theta)=\left(x_{\sigma}-y_{\sigma}\right)(\theta)$, para $\theta \in[-r, 0]$. Portanto,

$$
\sup _{t \in\left[\sigma-r, \sigma+\alpha_{1}\right]}|x(t)-y(t)| \leq \sup _{t \in\left[\sigma, \sigma+\alpha_{1}\right]}|x(t)-y(t)| .
$$

Aplicando o resultado acima na equação (1.12), segue que

$$
\sup _{t \in\left[\sigma-r, \sigma+\alpha_{1}\right]}|x(t)-y(t)| \leq \lambda \sup _{t \in\left[\sigma-r, \sigma+\alpha_{1}\right]}|x(t)-y(t)| .
$$

Dessa forma,

$$
(1-\lambda) \sup _{t \in\left[\sigma-r, \sigma+\alpha_{1}\right]}|x(t)-y(t)| \leq 0,
$$

o que implica que $x(t)=y(t)$ para todo $t \in\left[\sigma-r, \sigma+\alpha_{1}\right]$.

Se $\alpha_{1}=\alpha$ o teorema está demonstrado. Caso contrário, $\alpha_{1}<\alpha$, tome $\sigma^{\prime}=\sigma+\alpha_{1}$ e $\phi^{\prime}=x_{\sigma^{\prime}}=y_{\sigma^{\prime}}$. Nesse caso, $x$ e $y$ são soluções passando por $\left(\sigma^{\prime}, \phi^{\prime}\right)$ definidas em $\left[\sigma-r, \sigma+\alpha_{1}\right]$. De maneira análoga, é possível obter $\alpha_{2}>0$ de tal forma que $\alpha_{2} \leq \alpha_{1}$, $k \alpha_{2}<1$ e $x=y$ em $\left[\sigma-r, \sigma^{\prime}+\alpha_{2}\right]$.

Repetindo esse processo, obtém-se uma sequência $\left(\xi_{n}\right)$, onde $\xi_{n}=\sum_{i=1}^{n} \alpha_{1}$ e $x=y$ em $\left[\sigma-r, \sigma+\xi_{n}\right]$, para todo $n$.

A sequência $\left(\xi_{n}\right)$ é crescente e limitada por $\alpha$, logo existe $\alpha^{*}=\sup _{n \in \mathbb{N}} \xi_{n}$.

\section{Afirmação: $\alpha^{*}=\alpha$.}

Suponha que a afirmação não seja verdadeira, ou seja, $\alpha^{*}<\alpha$. Então, o maior intervalo em que $x=y$ é $\left[\sigma-r, \sigma-\alpha^{*}\right]$. Entretanto, tomando $\sigma^{*}=\sigma+\alpha^{*}$ e $\phi^{*}=x_{\sigma^{*}}=y_{\sigma^{*}}$, o processo aplicado acima garante que $x=y$ em $[\sigma-r, \sigma+\bar{\alpha}]$, contrariando o fato de $\left[\sigma-r, \sigma-\alpha^{*}\right]$ ser o maior intervalo em que as funções $x$ e $y$ coincidem.

Portanto existem uma única solução de (1.1) passando por $[\sigma-r, \sigma+\alpha]$. 



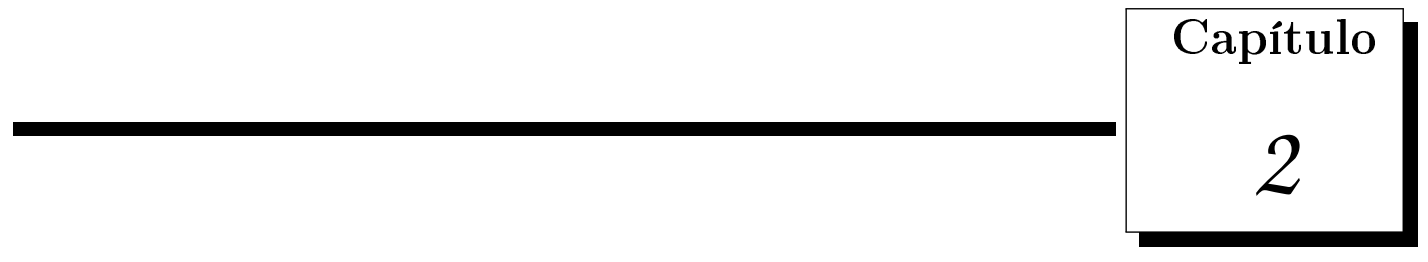

\section{Equação autônoma com linearidade} positiva

Neste capítulo, será estudado o comportamento global de soluções positivas da equação logística retardada com uma linearidade autônoma positiva. Serão estabelecidas condições suficientes para garantir a estabilidade global das soluções em torno do equilíbrio positivo. As técnicas desenvolvidas serão também utilizadas nos capítulos subsequentes, buscando estender o estudo a equações mais gerais.

\subsection{Equação Logística Retardada}

Considere a equação logística retardada:

$$
\dot{x}(t)=b(t) x(t)\left[1-L\left(x_{t}\right)\right]
$$

onde $L: C \rightarrow \mathbb{R}$ é um operador linear positivo. Dessa forma, ele é limitado e existe uma função não decrescente $\eta:[-r, 0] \rightarrow \mathbb{R}$ tal que

$$
L(\varphi)=\int_{-r}^{0} \varphi(\theta) d \eta(\theta), \quad \varphi \in C .
$$


Para $\varphi \in C$, tome o P.V.I. (2.1) com condição inicial

$$
x_{0}=\varphi \quad \operatorname{com} \varphi(\theta) \geq 0, \quad \theta \in[-r, 0) \quad \text { e } \quad \varphi(0)>0 .
$$

Além disso, considere as hipóteses adicionais (H1) e (H2), onde:

(H1) (i) $b: \mathbb{R} \rightarrow \mathbb{R}$ é uma função contínua e existem constantes $\beta_{0}$ e $\beta^{0}$ tais que $0<\beta_{0} \leq b(t) \leq \beta^{0}, \forall t \in \mathbb{R}$.

(ii) $L: C \rightarrow \mathbb{R}$ é um operador linear positivo $\operatorname{com} l:=\|L\|>0$.

(H2) Para toda $\varphi \in C$ tal que $|\varphi|_{C}=\varphi(0)>0$, segue que $L(\varphi)>0$.

Observação 1. A "ideia"da hipótese (H2) é que a função não descrescente $\eta:[-r, 0] \rightarrow \mathbb{R}$ tenha "mais relevância"perto de zero. Este é o caso em que L tem uma parte sem retardo que "domina" a parte com retardo. O exemplo a seguir ilustra essa afirmação.

Considere o operador

$$
L(\varphi)=b_{0} \varphi(0)+L_{0}(\varphi)
$$

onde $L_{0}: C \rightarrow \mathbb{R}$ é um operador linear positivo e $b_{0}>0$. Se $b_{0}>\left\|L_{0}\right\|$, então, para $|\varphi|_{C}=\varphi(0)>0$, tem-se que

$$
L(\varphi) \geq b_{0} \varphi(0)-\left\|L_{0}\right\||\varphi|_{C}>0
$$

isto é, (H2) é satisfeita.

No caso de um operador $L: C \rightarrow \mathbb{R}$ linear positivo, a hipótese (H2) estará satisfeita quando a seguinte condição for verdadeira:

$\left(\mathrm{H} 2^{*}\right)$ Existe uma constante $d \in(0,1)$ tal que se $\varphi \in C, \varphi(0)>0$ e $L(\varphi)=0$, então $\varphi(0) \leq d|\varphi|_{C}$

Suponha que a hipótese $(\mathrm{H} 2 *)$ está satisfeita. Para mostrar que (H2) é verdadeira, tome $\varphi \in C$ tal que $|\varphi|_{C}=\varphi(0)>0$ e suponha que $L(\varphi)=0$. Então,

$$
\varphi(0) \leq d|\varphi|_{C}=d \varphi(0),
$$


ou seja, $(1-d) \varphi(0) \geq 1$, mostrando que $d \leq 1$, o que é uma contradição. Logo, $L(\varphi)>0$.

Considere agora o operador

$$
L(\varphi)=\varphi(0)+\int_{-1}^{0} \varphi(\theta) d \theta
$$

que é um caso particular de (2.3). Dessa forma, (H2) está satisfeita.

Além disso, com este exemplo, é possível mostrar que $\left(\mathrm{H} 2^{*}\right)$ é "mais restritiva"que (H2). De fato, seja $\varepsilon \in(0,1), t_{0}=\frac{-2 \varepsilon}{2+\varepsilon}$ e $\varphi_{\varepsilon}:[-1,0] \rightarrow \mathbb{R}$ definida por

$$
\varphi_{\varepsilon}(x)=\left\{\begin{array}{l}
-1-\varepsilon, x \in\left[-1, t_{0}\right] \\
\frac{(2+\varepsilon)^{2} x}{2 \varepsilon}+1, x \in\left(t_{0}, 0\right]
\end{array}\right.
$$

Dessa forma, $\varphi_{\varepsilon}(0)=1>0$.

Afirmação $1:\left|\varphi_{\varepsilon}\right|_{C}=1+\varepsilon$.

Se $x \in\left[-1, t_{0}\right]$, então $\left|\varphi_{\varepsilon}\right|_{C}=1+\varepsilon$. Se $t_{0}<x \leq 0$, então

$$
t_{0} \frac{(2+\varepsilon)^{2}}{2 \varepsilon}<\frac{(2+\varepsilon)^{2}}{2 \varepsilon} x \leq 0
$$

ou seja,

$$
\left(\frac{-2 \varepsilon}{2+\varepsilon}\right) \frac{(2+\varepsilon)^{2}}{2 \varepsilon}<\frac{(2+\varepsilon)^{2}}{2 \varepsilon} x \leq 0,
$$

$\log 0$,

$$
-(2+\varepsilon)+1<\frac{(2+\varepsilon)^{2}}{2 \varepsilon} x+1 \leq 1,
$$

e, portanto, para $x \in\left(t_{0}, 0\right]$,

$$
-1-\varepsilon<\varphi_{\varepsilon}(x) \leq 1
$$

Dessa forma, conclui-se que $\left|\varphi_{\varepsilon}\right|_{C}=\max _{x \in[-1,0]}\left|\varphi_{\varepsilon}(x)\right|=1+\varepsilon$. 
Afirmação $2: L\left(\varphi_{\varepsilon}\right)=0$. De fato,

$$
\begin{aligned}
L\left(\varphi_{\varepsilon}\right) & =\varphi_{\varepsilon}(0)+\int_{-1}^{0} \varphi_{\varepsilon}(\theta) d \theta=1+\int_{-1}^{t_{0}}(-1-\varepsilon) d \theta+\int_{t_{0}}^{0}\left(\frac{(2+\varepsilon)^{2} \theta}{2 \varepsilon}+1\right) d \theta \\
& =1-\left(t_{0}+1\right)-\varepsilon\left(t_{0}+1\right)+\frac{(2+\varepsilon)^{2}}{2 \varepsilon}\left(-\frac{t_{0}^{2}}{2}\right)-t_{0} \\
& =t_{0}(-2-\varepsilon)-\varepsilon-\frac{(2+\varepsilon)^{2}}{4 \varepsilon} t_{0}^{2}=\left(\frac{-2 \varepsilon}{2+\varepsilon}\right)(-2-\varepsilon)-\varepsilon-\frac{(2+\varepsilon)^{2}}{4 \varepsilon} \frac{4 \varepsilon^{2}}{(2+\varepsilon)^{2}} \\
& =2 \varepsilon-\varepsilon-\varepsilon=0
\end{aligned}
$$

Para que a condição $\left(H 2^{*}\right)$ esteja satisfeita, deve existir $d \in(0,1)$ tal que $\mid \varphi_{\varepsilon}(0) \leq$ $d\left|\varphi_{\varepsilon}\right|_{C}$, ou seja, $1 \leq d(1+\varepsilon)$. Como $\varepsilon>0$ é arbitrário, $d \geq 1$, contradição.

Portanto vale (H2) mas não vale $(\mathrm{H} 2 *)$.

\subsection{Resultados importantes}

Teorema 2.1. Considere a equação (2.1) e suponha que (H1) esteja satisfeita. Defina $x^{*}=l^{-1}$ e considere o P.V.I. (2.1) com condição inicial (2.2) e solução $x(\varphi)(t)$.

Então valem:

(1) $x(\varphi)(t)$ é definida para $t \geq 0$, limitada em $[0, \infty)$ e limitada inferiormente longe do zero.

(2) Se $x(\varphi)(t)$ é solução não-oscilatória em torno de $x_{*}$, então $x(\varphi)(t) \rightarrow x_{*}$, quando $t \rightarrow \infty$.

\section{Prova:}

(1) Considere a equação (2.1) com a seguinte troca de variáveis: $y=\frac{x-x_{*}}{x_{*}}$.

Dessa forma,

$$
\left\{\begin{array}{l}
\dot{x}(t)=b(t) x(t)\left[1-L\left(x_{t}\right)\right] \\
y(t)=\frac{x(t)-x_{*}}{x_{*}}
\end{array}\right.
$$

Note que, como L é um operador linear, $L\left(x_{t}\right)=x_{*} L\left(y_{t}\right)+1$. Dessa forma,

$$
\dot{y}(t)=\frac{\dot{x}(t)}{x_{*}}=\frac{b(t) x(t)\left[1-L\left(x_{t}\right)\right]}{x_{*}}=-x_{*} b(t)(y(t)+1) L\left(y_{t}\right) .
$$


Equivalentemente,

$$
\dot{y}(t)=-x_{*} b(t)(y(t)+1) \int_{t-r}^{t} y(s) d \eta(s-t) .
$$

A hipótese $(\mathrm{H} 1)$ garante que $b(t) \leq \beta^{0}$ para todo $t \in \mathbb{R}$. Dessa forma, para $y>-1$ segue que $b(t) y>-\beta^{0}$. Além disso, para $y(t) \neq-1,(2.5)$ pode ser reescrita da seguinte forma:

$$
\frac{\dot{y}(t)}{1+y(t)}=-\int_{t-r}^{t} x_{*} b(t) y(s) d \eta(s-t)
$$

Integrando a expressão acima de 0 a t, obtém-se que

$$
\int_{0}^{t} \frac{y^{\prime}(\tau)}{1+y(\tau)} d(\tau)=-\int_{0}^{t} x_{*} b(\tau) \int_{\tau-r}^{\tau} y(\tau) d \eta(s-\tau) d \tau=-\int_{0}^{t} x_{*} b(\tau) L\left(y_{\tau}\right) d \tau .
$$

Assim,

$$
\ln (1+y(t))-\ln (1+y(0))=\int_{0}^{t} x_{*} b(\tau) L\left(y_{\tau}\right) d \tau
$$

$\log \mathrm{e}$

$$
1+y(t)=(1+y(0)) e^{-\int_{0}^{t} x_{*} b(\tau) L\left(y_{\tau}\right) d \tau}
$$

Portanto,

$$
y(t)=\left(1+\frac{\varphi(0)-x_{*}}{x_{*}}\right) e^{-\int_{0}^{t} x_{*} b(\tau) L\left(y_{\tau}\right) d \tau}-1,
$$

mostrando que $y(t)>-1, \forall t \geq 0$.

Note que foi considerado que $1+y(t) \neq 0$, o que é sempre verdade. De fato, suponha que exista $\bar{t}>0$ tal que $y(\widetilde{\varphi})(\bar{t})=-1$ e $y(\widetilde{\varphi})(\bar{t})>-1$ para $t<\bar{t}$. Assim, existe $\bar{M}>0$ tal que $|y(\widetilde{\phi})(\bar{t})| \leq \bar{M}$, quando $t \leq \bar{t}$.

Sendo assim, se $t \leq \bar{t}$ e $s \in[t-r, t]$, então $y(\widetilde{\phi})(\bar{t}) \leq \bar{M}$. Dessa forma

$$
\int_{t-r}^{t} y(s) d \eta(s-t) \leq \bar{M} \int_{t-r}^{t} d \eta(s-t)=\bar{M} \frac{1}{x_{*}}
$$

Portanto,

$$
-x_{*} b(t) \int_{t-r}^{t} y(s) d \eta(s-t) \geq-\bar{M} b(t) \geq-\bar{M} \beta^{0}, \forall t \leq \bar{t}
$$


e assim,

$$
y^{\prime}(t) \geq-(1+y(t)) \bar{M} \beta^{0}, \forall t \leq \bar{t} .
$$

Integrando a expressão acima de 0 a $\widetilde{t}$, com $\widetilde{t}<\bar{t}$ obtém-se que

$$
\ln (1+y(\widetilde{t}))-\ln (1-\widetilde{\phi}(0)) \geq-\bar{M} \beta^{0} \widetilde{t} .
$$

Logo,

$$
1+y(\widetilde{t}) \geq[1+\widetilde{\phi}(0)] e^{-\bar{M} \beta^{0} \widetilde{t}}, \quad \widetilde{t}<\bar{t}
$$

Portanto,

$$
\lim _{\widetilde{t} \rightarrow \bar{t}} y(\widetilde{t}) \geq(1+\widetilde{\phi}(0)) e^{-\bar{M} \beta^{0} \widetilde{t}}-1>-1,
$$

fornecendo uma contradição.

(2) Suponha que $x(\varphi)(t)$ é não oscilatória.

Sendo assim, existe $T>0$ tal que, se $t>T, y(t)$ não muda de sinal. Suponha, sem perda de generalidade, que $y(t)>0$, para $t>T$.

Considere $T_{1}=T+r$. Dessa forma,

$$
-y^{\prime}(t)=x_{*} b(t)(y(t)+1) \int_{t-r}^{t} y(s) d \eta(s-t), \text { para } t>T_{1}
$$

ou seja, $-y^{\prime}(t)>0, \forall t>T_{1}$, o que implica que $y$ é decrescente.

Assim, existe $c \geq 0$ tal que

$$
\lim _{t \rightarrow \infty} y(t)=c
$$

Suponha $c>0$. Então, para t suficientemente grande, existe $K$ tal que $y(t)>K>0$. Logo,

$$
\begin{aligned}
\left|\int_{0}^{t} \int_{\tau-r}^{\tau} x_{*} b(\tau) y(s) d \eta(s-\tau) d \tau\right| & >x_{*} \beta_{0} K \int_{0}^{t} \int_{\tau-r}^{\tau} d \eta(s-\tau) d \tau \\
& >\frac{x_{*} \beta_{0} K t}{x_{*}}=\beta_{0} K t \stackrel{t \rightarrow \infty}{\longrightarrow} \infty .
\end{aligned}
$$


Ou seja,

$$
\lim _{t \rightarrow \infty} \int_{0}^{t} \int_{\tau-r}^{\tau} x_{*} b(\tau) y(s) d \eta(s-\tau) d \tau=\infty .
$$

E como

$$
\ln \left(\frac{1+y(t)}{1+\widetilde{\varphi}(0)}\right)=-\int_{0}^{t} \int_{\tau-r}^{\tau} x_{*} b(\tau) y(s) d \eta(s-\tau) d \tau
$$

obtém-se que

$$
\lim _{t \rightarrow \infty} \ln \left(\frac{1+y(t)}{1+\widetilde{\varphi}(0)}\right)=-\infty
$$

o que implica que

$$
\lim _{t \rightarrow \infty} \frac{1+y(t)}{1+\widetilde{\varphi}(0)}=0
$$

permitindo conluir que

$$
\lim _{t \rightarrow \infty} y(t)=-1
$$

contradizendo o fato de $c>0$. Portanto,

$$
\lim _{t \rightarrow \infty} y(t)=0
$$

Para o próximo teorema, o seguinte lema será necessário:

Lema 2.1. Seja $0<\alpha<\frac{1}{2}$. Então, o sistema de desigualdades

$$
\left\{\begin{array}{l}
u \leq e^{v-\alpha v^{2}}-1 \\
v \leq 1-e^{-u-\alpha u^{2}}
\end{array}\right.
$$

tem uma única solução $(v, u)=(0,0)$ no primeiro quadrante.

Prova: Suponha que exista uma solução $\left(v_{0}, u_{0}\right) \neq(0,0)$ no primeiro quadrante. Logo, $u_{0}>0$ e $0<v_{0}<1$.

Defina as curvas

$$
\Gamma_{1}: u=e^{v-\alpha v^{2}}-1 \quad \text { e } \quad \Gamma_{2}: v=1-e^{-u-\alpha u^{2}} .
$$


Com relação à curva $\Gamma_{1}$, tem-se:

1. $\frac{\partial u}{\partial v}=e^{v-\alpha v^{2}}(1-2 \alpha v)$

- $\frac{\partial u}{\partial v}(0,0)=1$.

2. $\frac{\partial^{2} u}{\partial v^{2}}=e^{v-\alpha v^{2}}\left[(1-2 \alpha v)^{2}-2 \alpha\right]$

- $\frac{\partial^{2} u}{\partial v^{2}}(0,0)=1-2 \alpha$.

3. $\frac{\partial^{3} u}{\partial v^{3}}=e^{v-\alpha v^{2}}\left[(1-2 \alpha v)^{3}-6 \alpha(1-2 \alpha v)\right]$

- $\frac{\partial^{3} u}{\partial v^{3}}(0,0)=1-6 \alpha$

E com relação à $\Gamma_{2}$ :

1. $\frac{\partial u}{\partial v}=\frac{1}{(1+2 \alpha u) e^{-u-\alpha u^{2}}}$

- $\frac{\partial u}{\partial v}(0,0)=1$.

2. $\frac{\partial^{2} u}{\partial v^{2}}=\frac{\left(\frac{\partial u}{\partial v}\right)^{2}\left[(1+2 \alpha u)^{2}-2 \alpha\right]}{1+2 \alpha u}$

- $\frac{\partial^{2} u}{\partial v^{2}}(0,0)=1-2 \alpha$

3. $\frac{\partial^{3} u}{\partial v^{3}}=\frac{\left(\frac{\partial u}{\partial v}\right)^{3}\left(-8 \alpha^{3} u^{3}+18 \alpha^{3} u^{2}-12 \alpha^{2} u^{2}+20 \alpha^{2} u-6 \alpha u+6 \alpha-1\right)}{1+2 \alpha u}$

$$
\begin{aligned}
& +\frac{\frac{\partial u}{\partial v} \frac{\partial^{2} u}{\partial v^{2}}\left(12 \alpha^{2} u^{2}+12 \alpha u-6 \alpha+3\right)}{1+2 \alpha u} \\
& -\frac{\partial^{3} u}{\partial v^{3}}(0,0)=12 \alpha^{2}-6 \alpha+2 .
\end{aligned}
$$

Tudo isso permite ver que $\Gamma_{2}$ está acima de $\Gamma_{1}$ próximo de $(0,0)$.

Como $\left(v_{0}, u_{0}\right)$ é solução do primeiro quadrante, $\Gamma_{1}$ e $\Gamma_{2}$ devem se interceptar em algum ponto próximo de $(0,0)$. 
Considere $\left(v_{1}, u_{1}\right)$ ponto de intersecção de $\Gamma_{1}$ e $\Gamma_{2}$ tal que $v_{1}$ é o menor com essa propriedade. Assim, a inclinação de $\Gamma_{1}$ em $\left(v_{1}, u_{1}\right)$ não pode ser menor que a de $\Gamma_{2} \mathrm{em}$ $\left(v_{1}, u_{1}\right)$, isto é,

$$
\left.\frac{\partial u}{\partial v}\right|_{\Gamma_{1}}\left(v_{1}, u_{1}\right)=e^{v_{1}-\alpha v_{1}^{2}}\left(1-2 \alpha v_{1}\right) \geq \frac{1}{\left(1+2 \alpha u_{1}\right) e^{-u_{1}-\alpha u_{1}^{2}}}=\left.\frac{\partial u}{\partial v}\right|_{\Gamma_{2}}\left(v_{1}, u_{1}\right)
$$

ou seja,

$$
\left(1-2 \alpha v_{1}\right)\left(1+2 \alpha u_{1}\right) \geq e^{u_{1}-v_{1}+\alpha\left(u_{1}^{2}+v_{1}^{2}\right)} .
$$

Note que, $u_{1}>v_{1}$. Para mostrar que essa afirmação é verdadeira, defina

$$
\phi(x)=1-e^{-x-\alpha x^{2}}-x .
$$

Então, $\phi(0)=\phi^{\prime}(0)=0$ e $\phi^{\prime \prime}(x)=-e^{-x-\alpha x^{2}}\left[1+4 \alpha x+4 \alpha^{2} x^{2}-2 \alpha\right]<0$ para $x>0$. Dessa forma, $\phi^{\prime}(x)$ é descrescente quando $x>0$. E, como $\phi(0)=0$, conclui-se que $\phi(x)<0$ quando $x>0$. Em particular, $\phi\left(u_{1}\right)+u_{1}<u_{1}$. Além disso,

$$
\phi\left(u_{1}\right)+u_{1}=1-e^{-u_{1}-\alpha u_{1}^{2}}=v_{1} .
$$

Portanto, $u_{1}>v_{1}$.

É fato que $u_{1}-v_{1}+\alpha\left(u_{1}^{2}+v_{1}^{2}\right)>0$, já que $u_{1}>v_{1}$ e $\alpha>0$. Além disso, sabe-se que $e^{x}>1-x$ para $x>0$. Dessa forma

$$
e^{u_{1}-v_{1}+\alpha\left(u_{1}^{2}+v_{1}^{2}\right)}>u_{1}-v_{1}+\alpha\left(u_{1}^{2}+v_{1}^{2}\right)+1
$$

Substituindo o resultado acima em (2.6), segue que

$$
\left(1-2 \alpha v_{1}\right)\left(1+2 \alpha u_{1}\right)>u_{1}-v_{1}+\alpha\left(u_{1}^{2}+v_{1}^{2}\right)+1
$$

ou seja,

$$
(2 \alpha-1)\left(u_{1}-v_{1}\right)-4 \alpha^{2} u_{1} v_{1}>\alpha\left(u_{1}^{2}+v_{1}^{2}\right)
$$

Como $0<\alpha<\frac{1}{2}$, tem-se que $-4 \alpha^{2} u_{1} v_{1}>\alpha\left(u_{1}^{2}+v_{1}^{2}\right)$ e, então, $-4 \alpha u_{1} v_{1}>u_{1}^{2}+v_{1}^{2}$, o 
que é uma contradição.

Teorema 2.2. Considere a equação (2.1) e suponha que (H1) esteja satisfeita. Sendo $x(\varphi)(t)$ solução oscilatória em torno de $x_{*} e$

$$
\int_{t-r}^{t} b(\tau) d \tau \leq \frac{3}{2}
$$

para $t$ suficientemente grande, então $\lim _{t \rightarrow \infty} x(\varphi)(t)=x_{*}$

Prova: Considere $x(t)$ solução oscilatória de $(2.1)$ e a troca de variáveis $y=\frac{x-x_{*}}{x_{*}}$. Dessa forma, $y(t)$ é solução oscilatória de

$$
\dot{y}(t)=-x_{*} b(t)(y(t)+1) L\left(y_{t}\right) .
$$

Do Teorema 2.1, segue que

$$
\left\{\begin{array}{l}
y(t) \quad>-1, \forall t \geq 0 \\
y(t) \quad<e^{\frac{3}{2}}-1, \forall t \geq 0 .
\end{array}\right.
$$

Defina:

$$
u=\limsup _{t \rightarrow \infty} y(t) \quad e \quad v=-\liminf _{t \rightarrow \infty} y(t) .
$$

Dessa forma, $0 \leq v<1$ e $0 \leq u<\infty$.

A prova estará completa ao mostrar-se que $u=v=0$. Para isso, considere $0<\varepsilon<$ $1-v$. Então, existe $t_{0}=t_{0}(\varepsilon)$ de tal forma que

$$
-v-\epsilon<y(t)<u+\epsilon, \forall t \geq t_{0}-r
$$

e

$$
\int_{t-r}^{t} b(\tau) d \tau \leq \frac{3}{2}, \forall t \geq t_{0}-r
$$


A partir de (2.10), as seguintes desigualdades ficam satisfeitas:

$$
\begin{gathered}
\dot{y}(t) \leq b(t)(y(t)+1)(v+\epsilon), \forall t \geq t_{0}-r \\
\dot{y}(t) \geq-b(t)(y(t)+1)(u+\epsilon), \forall t \geq t_{0}-r .
\end{gathered}
$$

Considere a sequência crescente $\left\{t_{m}^{*}\right\}$ satisfazendo

$$
t_{m}^{*} \geq t_{0}, \quad \dot{y}\left(t_{m}^{*}\right)=0, \quad \lim _{m \rightarrow \infty} t_{m}^{*}=\infty \quad e \quad \lim _{t_{m}^{*} \rightarrow \infty} y\left(t_{m}^{*}\right)=u
$$

De (2.8) conclui-se que $L\left(y_{t_{m}^{*}}\right)=0$. Assim, existe $\xi_{m} \in\left(t_{m}^{*}-r, t_{m}^{*}\right)$ de tal forma que

$$
y\left(\xi_{m}\right)=0 \quad e \quad y(s)>0, s \in\left(\xi_{m}, t_{m}^{*}\right) .
$$

Integrando (2.12) de $s$ a $\xi_{m}$, segue que:

$$
\int_{s}^{\xi_{m}} \frac{\dot{y}(\tau)}{y(\tau)+1} d \tau \leq \int_{s}^{\xi_{m}} b(\tau)(v+\varepsilon) d \tau, \text { para } s \leq \xi_{m}
$$

Assim, para $s \leq \xi_{m}$,

$$
\ln \left(y\left(\xi_{m}\right)+1\right)-\ln (y(s)+1) \leq(v+\varepsilon) \int_{s}^{\xi_{m}} b(\tau) d \tau
$$

o que implica que

$$
\ln (y(s)+1) \geq-(v+\varepsilon) \int_{s}^{\xi_{m}} b(\tau) d \tau
$$

E, portanto,

$$
y(s) \geq e^{-(v+\varepsilon) \int_{s}^{\xi_{m}} b(\tau) d \tau}-1 .
$$

Em particular, para todo $t \in \mathbb{R}$,

$$
-b(t) y(s) \leq b(t)\left(1-e^{-(v+\varepsilon) \int_{s}^{\xi m} b(\tau) d \tau}\right) .
$$


Para $\xi_{m} \leq t \leq t_{m}^{*}$, tem-se que $t-r<\xi_{m}$ e

$$
\begin{aligned}
\dot{y}(t) & \leq-x_{*}(y(t)+1) \int_{t-r}^{\xi_{m}} b(\tau) y(s) d \eta(s-\tau) \\
& \leq x_{*} b(t)(y(t)+1) \int_{t-r}^{\xi_{m}}\left(1-e^{-(v+\varepsilon) \int_{s}^{\xi_{m}} b(\tau) d \tau}\right) d \eta(s-\tau) \\
& \leq x_{*} b(t)(y(t)+1)\left[\int_{t-r}^{t} d \eta(s-\tau)-e^{-(v+\varepsilon) \int_{t-r}^{\xi_{m}} b(\tau) d \tau} \int_{t-r}^{t} d \eta(s-\tau)\right] \\
& \leq b(t)(y(t)+1)\left[1-e^{-(v+\varepsilon) \int_{t-r}^{\xi_{m}} b(\tau) d \tau}\right]
\end{aligned}
$$

De (2.12) e (2.14), obtém-se que

$$
\frac{\dot{y}(t)}{y(t)+1} \leq \min \left\{b(t)(v+\varepsilon), b(t)\left[1-e^{-(v+\varepsilon) \int_{t-r}^{\xi_{m}} b(\tau) d \tau}\right]\right\}, \forall t \in\left[\xi_{m}, t_{m}^{*}\right]
$$

\section{Afirmação 1:}

$$
\ln \left(1+y\left(t_{m}^{*}\right) \leq v_{1}-\frac{1}{6} v_{1}^{2}\right.
$$

onde $v_{1}=v+\varepsilon$.

Esta afirmação estará provada ao se concluir o resultado para os dois seguintes casos:

Caso $1: \int_{\xi_{m}}^{t_{m}^{*}} b(\tau) d \tau \leq-\frac{\ln \left(1-v_{1}\right)}{v_{1}}$

Nas condições do Caso 1, de (2.15) segue que

$$
\begin{aligned}
\int_{\xi_{m}}^{t_{m}^{*}} \frac{\dot{y}(t)}{y(t)+1} d t & =\ln \left(y\left(t_{m}^{*}\right)+1\right) \leq \int_{\xi_{m}}^{t_{m}^{*}} b(t)\left[1-e^{-v_{1} \int_{t-r}^{\xi_{m}} b(\tau) d \tau}\right] d t \\
& =\int_{\xi_{m}}^{t_{m}^{*}} b(t)\left[1-e^{-v_{1}\left(\int_{t-r}^{t} b(\tau) d \tau-\int_{\xi_{m}}^{t} b(\tau) d \tau\right)}\right] d t \\
& \leq \int_{\xi_{m}}^{t_{m}^{*}} b(t)\left[1-e^{-\frac{3}{2}+v_{1} \int_{\xi_{m}}^{t} b(\tau) d \tau}\right] d t \\
& =\int_{\xi_{m}}^{t_{m}^{*}} b(t) d t-\int_{\xi_{m}}^{t_{m}^{*}} b(t) e^{-\frac{3}{2}+v_{1} \int_{\xi_{m}}^{t} b(\tau) d \tau} d t \\
& =\int_{\xi_{m}}^{t_{m}^{*}} b(t) d t-e^{-\frac{3}{2} v_{1}} \int_{\xi_{m}}^{t_{m}^{*}} b(t) e^{v_{1} \int_{\xi_{m}}^{t} b(\tau) d \tau} d t
\end{aligned}
$$

Utilizando a mudança de variáveis $u=\int_{\xi_{m}}^{t} b(\tau) d \tau, d u=b(t) d t$, obtém-se a solução da 
segunda integral do lado direito e, assim:

$$
\int_{\xi_{m}}^{t_{m}^{*}} b(t) e^{v_{1} \int_{\xi_{m}}^{t} b(\tau) d \tau} d t=\frac{1}{v_{1}}\left[e^{v_{1} \int_{\xi_{m}}^{t_{m}^{*}} b(\tau) d \tau}-1\right]
$$

Substituindo em (2.16), obtém-se que

$$
\begin{aligned}
\ln \left(y\left(t_{m}^{*}\right)+1\right) & \leq \int_{\xi_{m}}^{t_{m}^{*}} b(t) d t-\frac{1}{v_{1}} e^{\frac{3}{2} v_{1}}\left[e^{v_{1} \int_{\xi_{m}}^{t_{m}^{*}} b(\tau) d \tau}-1\right] \\
& =\int_{\xi_{m}}^{t_{m}^{*}} b(t) d t-\frac{1}{v_{1}} e^{-v_{1}\left(\frac{3}{2}-\int_{\xi_{m}}^{t_{m}^{*}} b(\tau) d \tau\right)}\left[1-e^{-v_{1} \int_{\xi_{m}}^{t_{m}^{*}} b(\tau) d \tau}\right] .
\end{aligned}
$$

Note que, para $\phi(x)=x-\frac{1}{v_{1}} e^{-v_{1}\left(\frac{3}{2}-x\right)}\left(1-e^{-v_{1} x}\right), \phi(x)$ é crescente para $0 \leq x \leq \frac{3}{2}$. De fato,

$$
\begin{aligned}
\dot{\phi}(x) & =1-\frac{1}{v_{1}} e^{-v_{1}\left(\frac{3}{2}-x\right)} v_{1}\left(1-e^{-v_{1} x}\right)-\frac{1}{v_{1}} e^{-v_{1}\left(\frac{3}{2}-x\right)}\left[-e^{-v_{1}}\left(-v_{1}\right)\right] \\
& =1-e^{-v_{1}\left(\frac{3}{2}-x\right)}+e^{-v_{1} x\left(\frac{3}{2}-x\right)-v_{1} x}-e^{-v_{1}\left(\frac{3}{2}-x\right)+v_{1} x} \\
& =1-e^{-v_{1}\left(\frac{3}{2}-x\right)} .
\end{aligned}
$$

Como $0 \leq x \leq \frac{3}{2}$ segue que $\frac{3}{2}-x \geq 0$. Além disso, $-v_{1}\left(\frac{3}{2}-x\right) \leq 0$, já que $v_{1}>0$. Dessa forma, $e^{-v_{1}\left(\frac{3}{2}-x\right)} \leq 1$. E, portanto, $\dot{\phi}(x)=1-e^{-v_{1}\left(\frac{3}{2}-x\right)} \geq 0$.

Então, como $\int_{\xi_{m}}^{t_{m}^{*}} b(\tau) d \tau \leq-\frac{\ln \left(1-v_{1}\right)}{v_{1}}$, segue que

$$
\begin{aligned}
\ln \left(1+y\left(t_{m}^{*}\right)\right) & \leq-\frac{\ln \left(1-v_{1}\right)}{v_{1}}-\frac{1}{v_{1}}\left[e^{-v_{1}\left(\frac{3}{2}+\frac{\ln \left(1-v_{1}\right)}{v_{1}}\right)}\right]\left[1-e^{\left.v_{1} \frac{\ln \left(1-v_{1}\right)}{v_{1}}\right]}\right. \\
& \leq-\frac{\ln \left(1-v_{1}\right)}{v_{1}}+\frac{1}{v_{1}}\left[v_{1}\left(\frac{3}{2}+\frac{\ln \left(1-v_{1}\right)}{v_{1}}\right)-1\right]\left[-1+1+v_{1}\right] \\
& \leq-\frac{\ln \left(1-v_{1}\right)}{v_{1}}+v_{1}\left(\frac{3}{2}+\frac{\ln \left(1-v_{1}\right)}{v_{1}}\right)-1 \\
& =\frac{3}{2} v_{1}-1-\ln \left(1-v_{1}\right)\left(\frac{1-v_{1}}{v_{1}}\right) .
\end{aligned}
$$

Afirmação 2: para $0<v_{1}<1$, vale:

$$
v+(1-v) \ln (1-v)=\int_{0}^{v}\left(\int_{0}^{x} \frac{1}{1-y} d y\right) d x
$$


De fato,

$$
\int_{0}^{x} \frac{1}{1-y} d y=-\ln (1-x)
$$

$\log \mathrm{o}$

$$
\int_{0}^{v} \int_{0}^{x} \frac{1}{1-y} d y d x=-\int_{0}^{v} \ln (1-x) d x
$$

Resolvendo a integral do lado direito, prova-se a Afirmação 2.

Além disso, para $0<v<1, \frac{1}{1-v}>1+v$. De fato, se existisse $v \in(0,1)$ tal que $\frac{1}{1-v} \leq 1+v, 1-v^{2} \geq 1$ deveria ser verdadeiro, o que não ocorre.

Dessa forma, da Afirmação 2 e do argumento acima decorre que

$$
\begin{aligned}
v+(1-v) \ln (1-v)=\int_{0}^{v}\left(\int_{0}^{x} \frac{d y}{1-y}\right) d x & >\int_{0}^{v} \int_{0}^{x}(1+y) d y d x \\
& =\frac{1}{2} v^{2}+\frac{1}{6} v^{3}
\end{aligned}
$$

Utilizando (2.19) com $v_{1}$ ao invés de $v$ e dividindo por $-v_{1}$, segue que

$$
-1-\frac{(1-v)}{v_{1}} \ln \left(1-v_{1}\right)<-\frac{1}{2} v_{1}-\frac{1}{6} v_{1}^{2}
$$

Somando-se $\frac{3}{2} v_{1}$ à desigualdade acima e aplicando o resultado obtido a (2.18) a Afirmação 1 está provada:

$$
\ln \left(1+y\left(t_{m}^{*}\right)\right) \leq \frac{3}{2} v_{1}-1-\frac{\left(1-v_{1}\right)}{v_{1}} \ln \left(1-v_{1}\right)<v_{1}-\frac{1}{6} v_{1}^{2}
$$

Caso $2:-\frac{\ln \left(1-v_{1}\right)}{v_{1}}<\int_{\xi_{m}}^{t_{m}^{*}} b(\tau) d \tau \leq \frac{3}{2}$

Falta ainda provar a Afirmação 1 nas condições do Caso 2. Para isso, escolha $\eta_{m} \in$ $\left[\xi_{m}, t_{m}^{*}\right]$ tal que

$$
\int_{\eta_{m}}^{t_{m}^{*}} b(\tau) d \tau=-\frac{\ln \left(1-v_{1}\right)}{v_{1}} .
$$


Para $t \in\left[\xi_{m}, \eta_{m}\right]$, de $(2.15)$ tem-se que

$$
\frac{y^{\prime}(t)}{1+y(t)} \leq b(t) v_{1}
$$

Logo,

$$
\int_{\xi_{m}}^{\eta_{m}} \frac{y^{\prime}(t)}{1+y(t)} d t \leq \int_{\xi_{m}}^{\eta_{m}} v_{1} b(t) d t
$$

Além disso, para $t \in\left[\eta_{m}, t_{m}^{*}\right]$,

$$
\frac{y^{\prime}(t)}{1+y(t)} \leq b(t)\left[1-e^{-v_{1} \int_{t-r}^{\xi_{m}} b(\tau) d \tau}\right]
$$

Logo,

$$
\int_{\eta_{m}}^{t_{m}^{*}} \frac{y^{\prime}(t)}{1+y(t)} d t \leq \int_{\eta_{m}}^{t_{m}^{*}} b(t)\left[1-e^{-v_{1} \int_{t-r}^{\xi m} b(\tau) d \tau}\right] d t
$$

Como

$$
\int_{\xi_{m}}^{\eta_{m}} \frac{y^{\prime}(t)}{1+y(t)} d t+\int_{\eta_{m}}^{t_{m}^{*}} \frac{y^{\prime}(t)}{1+y(t)} d t=\int_{\xi_{m}}^{t_{m}^{*}} \frac{y^{\prime}(t)}{1+y(t)} d t
$$

e

$$
\int_{\xi_{m}}^{t_{m}^{*}} \frac{y^{\prime}(t)}{1+y(t)} d t=\ln \left(1+y\left(t_{m}^{*}\right)\right)-\ln \left(1+y\left(\xi_{m}\right)\right)=\ln \left(1+y\left(t_{m}^{*}\right)\right)
$$

as desigualdades (2.21), (2.22), (2.23) e (2.24) permitem concluir que

$$
\begin{aligned}
\ln \left(1+y\left(t_{m}^{*}\right)\right) & \leq \int_{\xi_{m}}^{\eta_{m}} v_{1} b(t) d t+\int_{\eta_{m}}^{t_{m}^{*}} b(t)\left[1-e^{-v_{1} \int_{t-r}^{\xi_{m}} b(\tau) d \tau}\right] d t \\
& =v_{1} \int_{\xi_{m}}^{\eta_{m}} b(t) d t+\int_{\eta_{m}}^{t_{m}^{*}} b(t) d t-\int_{\eta_{m}}^{t_{m}^{*}} b(t) e^{-v_{1}\left[\int_{t-r}^{t} b(\tau) d \tau-\int_{\xi_{m}}^{t} b(\tau) d \tau\right]} d t
\end{aligned}
$$

Aplicando a segunda desigualdade do Caso 2 a (2.25), segue que

$$
\ln \left(1+y\left(t_{m}^{*}\right)\right) \leq v_{1} \int_{\xi_{m}}^{\eta_{m}} b(t) d t+\int_{\eta_{m}}^{t_{m}^{*}} b(t) d t-\int_{\eta_{m}}^{t_{m}^{*}} b(t) e^{-3 / 2 v_{1}+v_{1} \int_{\xi_{m}}^{t} b(\tau) d \tau} d t
$$

Utilizando a substituição $u=v_{1} \int_{\xi_{m}}^{t} b(\tau) d \tau, d u=v_{1} b(t) d t$, resolve-se a última integral 
do lado direito possibilitando obter:

$$
\int_{\eta_{m}}^{t_{m}^{*}} b(t) e^{-3 / 2 v_{1}+v_{1} \int_{\xi_{m}}^{t} b(\tau) d \tau} d t=\frac{e^{-3 / 2 v_{1}}}{v_{1}}\left[e^{v_{1} \int_{\xi_{m}}^{t_{m}^{*}} b(\tau) d \tau}-e^{v_{1} \int_{\xi_{m}}^{\eta_{m}} b(\tau) d \tau}\right]
$$

Voltando a (2.26),

$$
\begin{aligned}
\ln \left(1+y\left(t_{m}^{*}\right)\right) & \leq v_{1} \int_{\xi_{m}}^{\eta_{m}} b(t) d t+\int_{\eta_{m}}^{t_{m}^{*}} b(t) d t-\frac{e^{-3 / 2 v_{1}}}{v_{1}}\left[e^{v_{1} \int_{\xi_{m}}^{t_{m}^{*}} b(\tau) d \tau}-e^{v_{1} \int_{\xi_{m}}^{\eta_{m}} b(\tau) d \tau}\right] \\
& =v_{1} \int_{\xi_{m}}^{\eta_{m}} b(t) d t+\int_{\eta_{m}}^{t_{m}^{*}} b(t) d t-\frac{e^{-3 / 2 v_{1}}}{v_{1}}\left[e^{v_{1} \int_{\xi_{m}}^{t_{m}^{*}} b(\tau) d \tau}-e^{v_{1}\left[\int_{\xi m}^{t_{m}^{*}} b(\tau) d \tau-\int_{\eta_{m}}^{t_{m}^{*}} b(\tau) d \tau\right]}\right]
\end{aligned}
$$

Segundo (2.20), segue que

$$
\begin{aligned}
\ln \left(1+y\left(t_{m}^{*}\right)\right) & \leq v_{1} \int_{\xi_{m}}^{\eta_{m}} b(t) d t+\int_{\eta_{m}}^{t_{m}^{*}} b(t) d t-\frac{e^{-3 / 2 v_{1}}}{v_{1}}\left[e^{v_{1} \int_{\xi_{m}}^{t_{m}^{*}} b(\tau) d \tau}-e^{v_{1} \int_{\xi_{m}}^{t_{m}^{*}}} \cdot e^{\ln \left(1-v_{1}\right)}\right] \\
& =v_{1} \int_{\xi_{m}}^{\eta_{m}} b(t) d t+\int_{\eta_{m}}^{t_{m}^{*}} b(t) d t-\frac{e^{-3 / 2 v_{1}}}{v_{1}}\left[e^{v_{1} \int_{\xi_{m}}^{t_{m}^{*}} b(\tau) d \tau}-e^{v_{1} \int_{\xi_{m}}^{t_{m}^{*}}} \cdot\left(1-v_{1}\right)\right] \\
& =v_{1} \int_{\xi_{m}}^{\eta_{m}} b(t) d t+\int_{\eta_{m}}^{t_{m}^{*}} b(t) d t-\frac{e^{-3 / 2 v_{1}}}{v_{1}}\left[e^{v_{1} \int_{\xi_{m}}^{t_{m}^{*}} b(\tau) d \tau}\left(1-1+v_{1}\right)\right] \\
& =v_{1} \int_{\xi_{m}}^{\eta_{m}} b(t) d t+\int_{\eta_{m}}^{t_{m}^{*}} b(t) d t-e^{-\frac{3}{2} v_{1}+v_{1} \int_{\xi_{m}}^{t_{m}^{*}} b(\tau) d \tau} \\
& =v_{1} \int_{\xi_{m}}^{t_{m}^{*}} b(t) d t+\left(1-v_{1}\right) \int_{\eta_{m}}^{t_{m}^{*}} b(t) d t-e^{-\frac{3}{2} v_{1}+v_{1} \int_{\xi_{m}}^{t_{m}^{*}} b(\tau) d \tau} .
\end{aligned}
$$

Sabendo-se que $v_{1} \in(0,1), e^{-x}>1-x$ quando $x>0$ e utilizando a hipótese $\int_{\xi_{m}}^{t_{m}^{*}} b(\tau) d \tau=\frac{3}{2}$, segue que

$$
\begin{aligned}
\ln \left(1+y\left(t_{m}^{*}\right)\right) & \leq \frac{3}{2} v_{1}-\left(1-v_{1}\right)\left(\frac{\ln \left(1-v_{1}\right)}{v_{1}}\right)-1 \\
& =\frac{3}{2} v_{1}-\left(\frac{1-v_{1}}{v_{1}}\right) \ln \left(1-v_{1}\right)-1
\end{aligned}
$$

Da mesma forma como o Caso 1, conclui-se que

$$
\ln \left(1+y\left(t_{m}^{*}\right)\right) \leq v_{1}-\frac{1}{6} v_{1}^{2}
$$


o que prova a Afirmação 1.

Fazendo $m \rightarrow \infty$ e $\varepsilon \rightarrow 0$, segue que $v_{1} \rightarrow v$ e $y\left(t_{m}^{*}\right) \rightarrow u$. Logo,

$$
\ln (1+u) \leq v-\frac{1}{6} v^{2}
$$

Resta mostrar que

$$
-\ln (1-v) \leq u+\frac{1}{6} u^{2}
$$

Para isso, considere uma sequência $\left(s_{m}^{*}\right)$ satisfazendo

$$
s_{m}^{*} \leq t_{0}, \quad \dot{y}\left(s_{m}^{*}\right)=0, \quad \lim _{m \rightarrow \infty} s_{m}^{*}=\infty \quad e \quad \lim _{s_{m}^{*} \rightarrow \infty} y\left(s_{m}^{*}\right)=-v
$$

Analogamente ao que foi feito anteriormente, obtém-se uma sequência $\left\{\sigma_{m}\right\}$ de tal forma que $\sigma_{m} \in\left(s_{m}^{*}-r, s_{m}^{*}\right), y\left(\sigma_{m}\right)=0 \mathrm{e}$

$$
-\left(\frac{\dot{y}(t)}{1+y(t)}\right) \leq \min \left\{b(t) u_{1}, b(t)\left[e^{u_{1} \int_{t-r}^{\sigma_{m}} b(\tau) d \tau}-1\right]\right\}, \forall t \in\left[\sigma_{m}, s_{m}^{*}\right]
$$

\section{Afirmação 3:}

$$
-\ln \left(1+y\left(s_{m}^{*}\right)\right) \leq u_{1}+\frac{1}{6} u_{1}^{2}
$$

onde $u_{1}=u+\varepsilon$.

A prova da Afirmação 3 pode ser dividida em três etapas:

Caso $1: \int_{\sigma_{m}}^{s_{m}^{*}} b(\tau) d \tau \leq 1$

Nas condições do Caso 1 e considerando (2.29), segue que

$$
-\ln \left(1+y\left(s_{m}^{*}\right)\right)=-\int_{\sigma_{m}}^{s_{m}^{*}} \frac{\dot{y}(t)}{1+y(t)} d t \leq u_{1} \int_{\sigma_{m}}^{s_{m}^{*}} b(\tau) d \tau \leq u_{1} \leq u_{1}+\frac{1}{6} u_{1}^{2} .
$$

Caso $2: 1<\int_{\sigma_{m}}^{s_{m}^{*}} b(\tau) d \tau \leq \frac{3}{2}-\frac{\ln \left(1+u_{1}\right)}{u_{1}}$. 
A expressão em (2.29) garante que:

$$
\begin{aligned}
-\ln \left(1+y\left(s_{m}^{*}\right)\right) & =-\int_{\sigma_{m}}^{s_{m}^{*}} \frac{\dot{y}(t)}{1+y(t)} d t \leq u_{1} \int_{\sigma_{m}}^{s_{m}^{*}} b(\tau) d \tau \\
& \leq u_{1}\left(\frac{3}{2}-\frac{\ln \left(1+u_{1}\right)}{u_{1}}\right)=\frac{3}{2} u_{1}-\ln \left(1+u_{1}\right) .
\end{aligned}
$$

Note que, por hipótese, $1<\frac{3}{2}-\frac{\ln \left(1+u_{1}\right)}{u_{1}}$. Assim, $-\frac{1}{2}<-\frac{\ln \left(1+u_{1}\right)}{u_{1}}$. E, então $\frac{u_{1}}{2}>\ln \left(1+u_{1}\right), \log \mathrm{o}, u_{1}>2 \ln \left(1+u_{1}\right)>2$.

Se $u_{1} \geq 3: \frac{3}{2} u_{1}=u_{1}+\frac{u_{1}}{2} \leq u_{1}+\frac{u_{1}^{2}}{6}$. Portanto,

$$
\frac{3}{2} u_{1}-\ln \left(1+u_{1}\right) \leq u_{1}+\frac{u_{1}^{2}}{6}
$$

Caso contrário, se $2<u_{1}<3$, segue que

$$
\frac{u_{1}}{2}<\frac{3}{2}<\frac{2}{3}+\ln 3<\frac{2}{3}+\ln \left(u_{1}+1\right)<\frac{1}{6} u_{1}^{2}+\ln \left(u_{1}+1\right) .
$$

Logo,

$$
\frac{u_{1}}{2}-\ln \left(1+u_{1}\right)<\frac{1}{6} u_{1}^{2}
$$

e, portanto,

$$
\frac{3}{2} u_{1}-\ln \left(1+u_{1}\right)<u_{1}+\frac{1}{6} u_{1}^{2}
$$

Caso $3: \frac{3}{2}-\frac{\ln \left(1+u_{1}\right)}{u_{1}}<\int_{\sigma_{m}}^{s_{m}^{*}} b(\tau) d \tau \leq \frac{3}{2}$.

Seja $\eta_{m} \in\left[\sigma_{m}, s_{m}^{*}\right]$ tal que

$$
\frac{3}{2}-\frac{\ln \left(1+u_{1}\right)}{u_{1}}=\int_{\sigma_{m}}^{\eta_{m}} b(\tau) d \tau
$$

Para $t \in\left[\sigma_{m}, \eta_{m}\right]$, de (2.29) obtém-se que

$$
\frac{y^{\prime}(t)}{1+y(t)} \leq b(t) u_{1}
$$


Logo,

$$
\int_{\xi_{m}}^{\eta_{m}} \frac{y^{\prime}(t)}{1+y(t)} d t \leq u_{1} \int_{\xi_{m}}^{\eta_{m}} b(t) d t
$$

Além disso, para $t \in\left[\eta_{m}, s_{m}^{*}\right]$,

$$
\frac{y^{\prime}(t)}{1+y(t)} \leq b(t)\left[e^{u_{1} \int_{t-r}^{\sigma_{m}} b(\tau) d \tau}-1\right] .
$$

Logo,

$$
\int_{\eta_{m}}^{s_{m}^{*}} \frac{y^{\prime}(t)}{1+y(t)} d t \leq \int_{\eta_{m}}^{s_{m}^{*}} b(t)\left[e^{u_{1} \int_{t-r}^{\sigma_{m}} b(\tau) d \tau}-1\right] d t
$$

Note que

$$
\int_{\sigma_{m}}^{\eta_{m}} \frac{y^{\prime}(t)}{1+y(t)} d t+\int_{\eta_{m}}^{s_{m}^{*}} \frac{y^{\prime}(t)}{1+y(t)} d t=\int_{\sigma_{m}}^{s_{m}^{*}} \frac{y^{\prime}(t)}{1+y(t)} d t
$$

$\mathrm{e}$

$$
-\int_{\sigma_{m}}^{s_{m}^{*}} \frac{y^{\prime}(t)}{1+y(t)} d t=-\ln \left(1+y\left(s_{m}^{*}\right)\right)+\ln \left(1+y\left(\sigma_{m}\right)\right)=-\ln \left(1+y\left(s_{m}^{*}\right)\right)
$$

Dessa forma, as desigualdades (2.31), (2.32), (2.33) e (2.34) mostram que

$$
-\ln \left(1+y\left(s_{m}^{*}\right)\right) \leq \int_{\eta_{m}}^{s_{m}^{*}} b(t) e^{u_{1} \int_{t-r}^{\sigma_{m}} b \tau d \tau} d t+u_{1} \int_{\sigma_{m}}^{\eta_{m}} b(t) d t-\int_{\eta_{m}}^{s_{m}^{*}} b(t) d t
$$

Além disso, utilizando (2.30), obtém-se que

$$
\begin{aligned}
-\ln \left(1+y\left(s_{m}^{*}\right)\right) & \leq \int_{\eta_{m}}^{s_{m}^{*}} b(t) e^{u_{1}\left(\int_{t-r}^{t} b(\tau) d \tau-\int_{\sigma_{m}}^{t} b(\tau) d \tau\right)} d t \\
& +u_{1}\left(\frac{3}{2}-\frac{\ln \left(1+u_{1}\right)}{u_{1}}\right)-\int_{\eta_{m}}^{s_{m}^{*}} b(t) d t .
\end{aligned}
$$


De acordo com (2.7), segue que

$$
\begin{aligned}
-\ln \left(1+y\left(s_{m}^{*}\right)\right) & \leq e^{\frac{3}{2} u_{1}} \int_{\eta_{m}}^{s_{m}^{*}} b(t) e^{-u_{1} \int_{\sigma_{m}}^{t} d(\tau) d \tau} d t \\
& +u_{1}\left(\frac{3}{2}-\frac{\ln \left(1+u_{1}\right)}{u_{1}}\right)-\int_{\eta_{m}}^{s_{m}^{*}} b(t) d t .
\end{aligned}
$$

A solução da primeira integral do lado direito é obtida a partir da substituição $v=$ $-u_{1} \int_{\sigma_{m}}^{t} b(\tau) d \tau, d v=-u_{1} b(t) d t$ e, dessa forma,

$$
\int_{\eta_{m}}^{s_{m}^{*}} b(t) e^{-u_{1} \int_{\sigma_{m}}^{t} d(\tau) d \tau} d t=-\frac{1}{u_{1}}\left[e^{-u_{1} \int_{\sigma_{m}}^{s_{m}^{*}} b(\tau) d \tau}-e^{-u_{1} \int_{\sigma_{m}}^{\eta_{m}} b(\tau) d \tau}\right] .
$$

Voltando a (2.37),

$$
\begin{aligned}
-\ln \left(1+y\left(s_{m}^{*}\right)\right) & \leq-\frac{e^{\frac{3}{2} u_{1}}}{u_{1}}\left[e^{-u_{1} \int_{\sigma_{m}}^{s_{m}^{*}} b(\tau) d \tau}-e^{-u_{1} \int_{\sigma_{m}}^{\eta_{m}} b(\tau) d \tau}\right]+u_{1}\left(\frac{3}{2}-\frac{\ln \left(1+u_{1}\right)}{u_{1}}\right)-\int_{\eta_{m}}^{s_{m}^{*}} b(t) d t \\
& =\frac{e^{\frac{3}{2} u_{1}}}{u_{1}}\left[e^{-u_{1} \int_{\sigma_{m}}^{\eta_{m}} b(\tau) d \tau}-e^{-u_{1} \int_{\sigma_{m}}^{s_{m}^{*}} b(\tau) d \tau}\right]+u_{1}\left(\frac{3}{2}-\frac{\ln \left(1+u_{1}\right)}{u_{1}}\right)-\int_{\eta_{m}}^{s_{m}^{*}} b(t) d t .
\end{aligned}
$$

De (2.30), segue que

$$
\begin{aligned}
-\ln \left(1+y\left(s_{m}^{*}\right)\right) & \leq \frac{e^{\frac{3}{2} u_{1}}}{u_{1}}\left[e^{-\frac{3}{2} u_{1}+\ln \left(1+u_{1}\right)}-e^{-u_{1} \int_{\sigma_{m}}^{s_{m}^{*}} b(\tau) d \tau}\right]+u_{1}\left(\frac{3}{2}-\frac{\ln \left(1+u_{1}\right)}{u_{1}}\right)-\int_{\eta_{m}}^{s_{m}^{*}} b(t) d t \\
& =\frac{1}{u_{1}}\left[1+u_{1}-e^{\frac{3}{2} u_{1}-u_{1} \int_{\sigma_{m}}^{s_{m}^{*}} b(\tau) d \tau}\right]+u_{1}\left(\frac{3}{2}-\frac{\ln \left(1+u_{1}\right)}{u_{1}}\right)-\int_{\eta_{m}}^{s_{m}^{*}} b(t) d t .
\end{aligned}
$$

Utilizando o fato que $e^{-x}<1-x$ quando $x>0$ na desigualdade acima, segue que

$$
\begin{aligned}
-\ln \left(1+y\left(s_{m}^{*}\right)\right) & \leq \frac{1}{u_{1}}\left[1+u_{1}-1-\frac{3}{2} u_{1}+u_{1} \int_{\sigma_{m}}^{s_{m}^{*}} b(\tau) d \tau\right]+u_{1}\left(\frac{3}{2}-\frac{\ln \left(1+u_{1}\right)}{u_{1}}\right)-\int_{\eta_{m}}^{s_{m}^{*}} b(t) d t \\
& =1-\frac{3}{2}+\int_{\eta_{m}}^{s_{m}^{*}} b(t) d t+u_{1}\left(\frac{3}{2}-\frac{\ln \left(1+u_{1}\right)}{u_{1}}\right)-\int_{\eta_{m}}^{s_{m}^{*}} b(t) d t .
\end{aligned}
$$


Aplicando (2.30) novamente,

$$
\begin{aligned}
-\ln \left(1+y\left(s_{m}^{*}\right)\right) & \leq-\frac{1}{2}+\frac{3}{2}-\frac{\ln \left(1+u_{1}\right)}{u_{1}}+u_{1}\left(\frac{3}{2}-\frac{\ln \left(1+u_{1}\right)}{u_{1}}\right) \\
& =\frac{3}{2} u_{1}+1-\left(\frac{1+u_{1}}{u_{1}}\right) \ln \left(1+u_{1}\right) .
\end{aligned}
$$

Afirmação 4: para $u>0$, segue que

$$
(1+u) \ln (1+u)-u=\int_{0}^{u}\left(\int_{0}^{x} \frac{d y}{1+y}\right) d x
$$

De fato,

$$
\int_{0}^{x} \frac{d y}{1+y}=\ln (1+x)
$$

$\log \mathrm{e}$

$$
\int_{0}^{u}\left(\int_{0}^{x} \frac{d y}{1+y}\right) d x=\int_{0}^{u} \ln (1+x) d x
$$

Resolvendo a integral do lado direito, prova-se a Afirmação 4.

Além disso, para $u>0$ tem que $\frac{1}{1+u}>(1-u)$. De fato, se existisse $u>0$ tal que $\frac{1}{1+u} \leq(1-u), 1 \leq 1-u^{2}$ deveria ser verdadeiro, o que não ocorre.

Dessa forma, da Afirmação 4 e do argumento acima decorre que

$$
\begin{aligned}
(1+u) \ln (1+u)-u=\int_{0}^{u}\left(\int_{0}^{x} \frac{d y}{1+y}\right) d x & >\int_{0}^{u} \int_{0}^{x}(1-y) d y d x \\
& =\frac{u^{2}}{2}-\frac{u^{3}}{6} .
\end{aligned}
$$

Utilizando (2.39) com $u_{1}$ no lugar de $u$ e dividindo os dois lados por $-u_{1}$ :

$$
1-\left(\frac{1+u_{1}}{u_{1}}\right) \ln \left(1+u_{1}\right)<-\frac{u_{1}}{2}+\frac{u_{1}^{2}}{6}
$$

Somando-se $\frac{3}{2} u_{1}$ à desigualdade acima e aplicando o resultado obtido a (2.38) a Afirmação 3 está provada:

$$
-\ln \left(1+y\left(s_{m}^{*}\right)\right) \leq \frac{3}{2} u_{1}+1-\left(\frac{1+u_{1}}{u_{1}}\right) \ln \left(1+u_{1}\right)<\frac{u_{1}}{2}+\frac{u_{1}^{2}}{6}
$$


Fazendo $\varepsilon \rightarrow \infty$ e $m \rightarrow \infty$, conclui-se que

$$
-\ln (1-v) \leq u+\frac{1}{6} u^{2}
$$

E, com $\alpha=\frac{1}{6}$, pelo Lema 2.1, a prova do Teorema 2.2 está finalizada.

Teorema 2.3. Assuma que (H1) e (H2) estejam satisfeitas. Então, as soluções $x(t)$ do P.V.I (2.1), (2.2) satisfazem:

$$
\lim _{t \rightarrow \infty} x(t)=x_{*},
$$

onde $x_{*}=l^{-1}$.

Prova: O caso em que $x(t)$ é não oscilatória foi provado no Teorema 2.1. Dessa forma, basta analisar o caso em que $x(t)$ é solução oscilatória em torno de $x_{*}$.

Considere a mudança de variáveis $y=\frac{x-x_{*}}{x_{*}}$. Então, $y(t)$ é solução de (2.8).

Defina

$$
\liminf _{t \rightarrow \infty} y(t)=-v \quad e \quad \limsup _{t \rightarrow \infty} y(t)=u
$$

Então, $0 \leq v<1$ e $0 \leq u<\infty$.

Tome $\varepsilon>0$ e $T>0$ tais que

$$
-v-\varepsilon \leq y(t) \leq u+\varepsilon, \forall t>T \text {. }
$$

Para provar o teorema, basta mostrar que $u=v=0$.

Caso 1: $v \leq u$.

Considere $\left\{t_{n}\right\} \subset \mathbb{R}$ uma sequência tal que

$$
t_{n}-r>T, \quad \dot{y}\left(t_{n}\right)=0, \quad y\left(t_{n}\right)>0 . \quad \lim _{n \rightarrow \infty} t_{n}=\infty \quad e \quad \lim _{t \rightarrow \infty} y\left(t_{n}\right)=u .
$$

De acordo com (2.8), $L\left(y_{t_{n}}\right)=0$.

Note que, de acordo com o Teorema 2.1 sabe-se que y é limitada. Além disso, (2.8) indica que $y$ é limitada. Logo, $y$ é Lipschitz. Mais ainda, $y_{t_{n}}$ é equicontínua e uniformemente 
limitada. Sendo assim, o Teorema de Ascoli garante que $y_{t_{n}}$ possui uma subsequência, aqui representada com a mesma notação, tal que $y_{t_{n}}$ converge para alguma $\varphi \in C$

Como $L\left(y_{t_{n}}\right)=0$, para todo $t_{n}$, segue que $L(\varphi)=0$. Por outro lado, a equação $(2.40)$ juntamento com o fato de que $v \leq u$ implicam que

$$
\left|y_{t_{n}}\right|_{C}=\sup _{s \in[0, \infty)}\left|y_{t_{n}}(s)\right| \leq u+\varepsilon
$$

Como $\varepsilon$ é arbitrário, fazendo $n \rightarrow \infty$ segue que

$$
|\varphi|_{C} \leq u=\varphi(0)
$$

Da hipótese (H2) conclui-se que $u=v=0$.

Caso 2: $v \geq u$.

A prova é análoga à do Caso 1 . Basta considerar uma sequência $\left\{s_{n}\right\} \subset \mathbb{R}$ tal que

$$
s_{n}-r>T, \quad \dot{y}\left(s_{n}\right)=0, \quad y\left(s_{n}\right)<0, \quad \lim _{n \rightarrow \infty} s_{n}=\infty \quad e \quad \lim _{t \rightarrow \infty} y\left(s_{n}\right)=-v .
$$

Lema 2.2. Considere a equação (2.1), com $b: \mathbb{R} \rightarrow \mathbb{R}$ uma função contínua positiva $e$ suponha que as hipóteses $(H 1)(i i)$ e (H2) estejam satisfeitas. Defina $x_{*}=l^{-1}$ e considere $x(t)=x(\varphi)(t)$ solução de (2.1). Suponha que $x(t)$ está definida em $I=(a, \infty)$, com $a \in \mathbb{R}$ ou $a=-\infty$ e que existam $K>0, t_{0}>a+r$ tais que $x(t)$ satisfaz:

$$
\left|x(t)-x_{*}\right| \leq K, \text { para } t \in\left(a, t_{0}\right] .
$$

Então,

$$
\left|x(t)-x_{*}\right| \leq K, \text { para } t \in\left[t_{0}, \infty\right)
$$

Prova : Suponha que exista $t_{1}>t_{0}$ tal que $\left|x\left(t_{1}\right)-x_{*}\right|>K$. Defina

$$
T=\min \left\{t \in\left[t_{0}, t_{1}\right]:\left|x(t)-x_{*}\right|=\sup _{t_{0} \leq s \leq t_{1}}\left|x(s)-x_{*}\right|\right\}
$$


Então $T \in\left(t_{0}, t_{1}\right]$ e $\left|x(s)-x_{*}\right|<\left|x(T)-x_{*}\right|, \forall s \in\left(t_{0}, T\right)$. Logo,

$$
\left|x(s)-x_{*}\right| \leq K<\left|x(T)-x_{*}\right|, \forall s \in\left(a, t_{0}\right] .
$$

Em particular,

$$
\left|x(s)-x_{*}\right|<\left|x(T)-x_{*}\right|, \forall s \in(a, T) .
$$

Se $x(T)-x_{*}>0$, então $\left|x(s)-x_{*}\right|<x(T)-x_{*}, \forall s \in(a, T)$, o que implica que,

$$
x(s)-x_{*}<x(T)-x_{*}, \forall s \in(a, T) .
$$

Logo, $x(s)<x(T), \forall s \in(a, T)$, mostrando que $\dot{x}(T) \geq 0$. Por outro lado, (H2) implica que

$$
\dot{x}(T)=-b(T) x(T) L\left(x_{T}-x_{*}\right)<0,
$$

gerando uma contradição.

Se $x(T)-x_{*}<0$, então $\left|x(s)-x_{*}\right|<x_{*}-x(T), \forall s \in(a, T)$, o que implica que,

$$
x_{*}-x(s)<x_{*}-x(T), \forall s \in(a, T) .
$$

Logo, $x(s)>x(T), \forall s \in(a, T)$, mostrando que $\dot{x}(T) \leq 0$. Por outro lado, $(H 2)$ implica que

$$
\dot{x}(T)=-b(T) x(T) L\left(x_{T}-x_{*}\right)>0,
$$

fornecendo outra contradição.

Sendo assim, conclui-se que

$$
\left|x(t)-x_{*}\right| \leq K, \forall t \in\left[t_{0}, \infty\right)
$$

Lema 2.3. Seja $x_{*}=l_{1}$. Assuma que $(H 1)$ e (H2) estejam satisfeitas. Se $x(t)=x(\varphi)(t)$ for uma solução global de (2.1) que é limitada em $(-\infty, \infty)$ e limitada inferiormente longe 
do zero, então

$$
\lim _{t \rightarrow \infty} x(t)=x_{*}
$$

Prova : Considere a mudança de variáveis $y=\frac{x-x_{*}}{x_{*}}$. Então $y(t)$ é solução de $(2.8)$ em $\mathbb{R}$ com

$$
-1<m \leq y(t) \leq M, \forall t \in \mathbb{R}
$$

Se $y(t)<0$ para todo $t \in\left(-\infty, T_{0}\right]$ e algum $T_{0} \in \mathbb{R}$, como L é positivo, segue que $y(t)$ é não decrescente em $\left(-\infty, T_{0}\right]$ e, assim, existe c tal que

$$
c=\lim _{t \rightarrow-\infty} y(t)<0
$$

Para $t \leq T_{1},-T_{1}>0$ suficientemente grande, segue que $L\left(y_{t}\right) \leq \frac{1}{2} l c$ e, assim,

$$
\dot{y}(t) \geq-\frac{1}{2}(1+y(t)) \beta_{0} c .
$$

Integrando a expressão acima de $t$ a $T_{1}$,

$$
\ln \left(1+y\left(T_{1}\right)\right)-\ln (1+y(t)) \geq-\frac{1}{2} \beta_{0} c\left(T_{1}-t\right), t \leq T_{1}
$$

o que implica que

$$
1+y\left(T_{1}\right) \geq e^{-\frac{1}{2} \beta_{0} c\left(T_{1}-t\right)}(1+y(t)), t \leq T_{1}
$$

Note que o lado direito tende a $\infty$ quando $t \rightarrow \infty$, contradizendo o fato de $y$ ser limitada.

O caso em que $y(t)>0, t \in\left(\infty, T_{0}\right]$ para algum $T_{0} \in \mathbb{R}$ também não ocorre.

Assim, $y(t)$ é oscilatória perto de $-\infty$, isto é, $y(t)$ é oscilatória.

A prova que $\lim _{t \rightarrow-\infty} y(t)=0$ é análoga à do Teorema 2.3, bastando substituir a função $y(t)$ pela $z(t)=y(-t)$.

Teorema 2.4. Assuma que $(H 1)$ e (H2) estejam satisfeitas. Então, a única solução de (2.1) que é definida em $\mathbb{R}$, limitada e limitada inferiormente longe do zero é a solução 
constante $x(t)=x_{*}$, onde $x_{*}=l^{-1}$.

Prova : Seja $x(t)$ solução global de (2.1) que é limitada iferiormente longe do zero e limitada.

Segundo o Lema 2.3, tem-se $\lim _{t \rightarrow \infty} x(t)=x_{*}$.

Assim, para $\varepsilon>0$ arbitrário existe $t_{0}=t_{0}(\varepsilon)$ tal que

$$
\left|x(t)-x_{*}\right| \leq \varepsilon, \forall t \geq t_{0}
$$

Por outro lado, o Lema 2.2 garante que

$$
\left|x(t)-x_{*}\right| \leq \varepsilon, \forall t \in \mathbb{R}
$$

E, portanto,

$$
\lim _{t \rightarrow \infty} x(t)=x_{*} .
$$

Corolário 2.2.1. Considere a equação diferencial funcional escalar autônoma,

$$
\dot{x}(t)=b_{0} x(t)\left[1-L\left(x_{t}\right)\right],
$$

onde $b_{0}>0$ e $L: C \rightarrow \mathbb{R}$ é um operador linear positivo não nulo. Assuma que L satisfaz (H2).

Então, todas as soluções $x(\varphi)(t)$ de (2.41) com condição inicial $\varphi$ como em (2.2), tendem $a x_{*}$ quando $t$ tende a infinito, onde $x_{*}=l^{-1}$.

Além disso, $x_{*}$ é a única solução global de (2.41) que é limitada inferiormente longe do zero e limitada em $\mathbb{R}$. 


\subsection{Exemplo}

Exemplo 1. Considere a seguinte equação diferencial com retardo escalar, com um número finito de retardos

$$
\dot{x}(t)=x(t)\left[\alpha-b_{0} x(t)-\sum_{i=1}^{n} b_{i} x\left(t-r_{i}\right)\right],
$$

onde todas as constantes $\alpha, b_{i}, r_{i}, i=0, \ldots, n$, são positivas.

Se $b_{0}>\sum_{i=1}^{n} b_{i}$ então (H1) e (H2*) estão satisfeitas e $x_{*}=\alpha\left(\sum_{i=0}^{n} b_{i}\right)^{-1}$ é um equilíbrio globalmente estável.

Do Teorema 2.4, $x_{*}$ é a única solução global de (2.42) que é limitada e limitada inferiormente longe do zero. 



\title{
Capítulo
}

\section{Equação não autônoma com linearidade}

\author{
positiva
}

Este capítulo destina-se ao estudo da equação logística retardada com uma linearidade não autônoma. Considerando-se certas condições, será mostrado que todas as soluções positivas dessa equação tendem ao equilíbrio positivo $x_{*}$ quando t tende ao infinito.

\subsection{Equação Logística Retardada com uma linearidade}

\section{não autônoma}

Considere a Equação Logística Não Autônoma e retardada

$$
\dot{x}(t)=b(t) x(t)\left[a(t)-L\left(t, x_{t}\right)\right] .
$$

Para $\varphi \in C$, considere o P.V.I. (3.1) com condição inicial

$$
x_{0}=\varphi \quad \operatorname{com} \varphi(\theta) \geq 0, \quad \theta \in[-r, 0) \quad \text { e } \quad \varphi(0)>0 .
$$

Ao longo deste capítulo, também serão consideradas as seguintes hipóteses:

(H1) (i) $b: \mathbb{R} \rightarrow \mathbb{R}$ é uma função contínua e existem constantes $\beta_{0}$ e $\beta^{0}$ tais que 


$$
0<\beta_{0} \leq b(t) \leq \beta^{0}, \forall t \in \mathbb{R} .
$$

(ii) $L: C \rightarrow \mathbb{R}$ é um operador linear positivo $\operatorname{com} l:=\|L\|>0$.

(H2) Para toda $\varphi \in C$ tal que $|\varphi|_{C}=\varphi(0)>0$, segue que $L(\varphi)>0$.

$\left(\mathrm{H} 2^{*}\right)$ Existe uma constante $d \in(0,1)$ tal que se $\varphi \in C, \varphi(0)>0$ e $L(\varphi)=0$, então $\varphi(0) \leq d|\varphi|_{C}$

(A1) $a: \mathbb{R} \rightarrow \mathbb{R}$ e $b: \mathbb{R} \rightarrow \mathbb{R}$ funções contínuas e existem constantes $\beta_{0}$, $\beta^{0}$ tais que

$$
0<\beta_{0} \leq b(t) \leq \beta^{0}, \quad \forall t \in \mathbb{R}
$$

(A2) O operador $L(t, \cdot)$ é linear e positivo para todo $t \in \mathbb{R}$.

(A3) Para todo $M>0$, a função

$$
(t, \varphi) \mapsto a(t)-L(t, \varphi)
$$

é uniformemente limitada para $(t, \varphi) \in \mathbb{R} \times C,|\varphi|_{C} \leq M$.

(A4) Existe um $\alpha_{0}>0$ tal que

$$
L(t, 1)=\|L(t, .)\| \geq \alpha_{0}, \forall t \in \mathbb{R} .
$$

(A5) Para toda $\varphi \in C$, existe o $\lim _{t \rightarrow \infty} L(t, \varphi)$ e é definido por

$$
\lim _{t \rightarrow \infty} L(t, \varphi):=L(\varphi)
$$

Observação 2. A hipótese (A3) leva à conclusão de que a(t) é necessariamente limitada em $\mathbb{R}, j a ́$ que a $(t)$ pode ser vista como:

$$
a(t)=a(t)-L(t, 0)
$$

Sendo assim, pode-se dizer que $|a(t)| \leq M_{a}$, para algum $M_{a}>0$. 
Além disso, (A3) também garante a limitação uniforme de $L(t, \cdot)$ para todo $t \in \mathbb{R}$. Basta observar que

$$
L(t, \varphi)=a(t)-(a(t)-L(t, \varphi))
$$

\subsection{Resultados importantes}

Lema 3.1. Assumindo verdadeiras as hipóteses $(A 1)-(A 3)$, obtem-se que a solução $x(\varphi)(t)$ de (3.1), (3.2) é positiva e definida em $[0, \infty)$.

Prova: Sendo $x(t)=x(\varphi)(t)$ solução do P.V.I. (3.1), (3.2) e considerando a troca de variáveis: $y(t)=\frac{x(t)-x_{*}}{x_{*}}, y(t)$ se torna solução de

$$
\dot{y}(t)=b(t)(y(t)+1)\left[a(t)-x_{*} L\left(t, y_{t}\right)-x_{*} L(t, 1)\right]
$$

Integrando (3.3) de 0 a t:

$$
\int_{0}^{t} \frac{\dot{y}(s)}{y(s)+1} d s=\int_{0}^{t} b(s)\left[a(s)-x_{*} L\left(s, y_{s}\right)-x_{*} L(s, 1)\right] d s .
$$

Resolvendo-se o lado esquerdo da expressão acima, obtém-se que

$$
\ln (y(t)+1)-\ln (y(0)+1)=\int_{0}^{t} b(s)\left[a(s)-x_{*} L\left(s, y_{s}\right)-x_{*} L(s, 1)\right] d s
$$

$\log \mathrm{o}$

$$
y(t)=\left(1-\frac{\varphi(0)-x_{*}}{x_{*}}\right) e^{\int_{0}^{t} b(s)\left[a(s)-x_{*} L\left(s, y_{s}\right)-x_{*} L(s, 1)\right] d s}-1 .
$$

ou seja, $y(t)>-1$, para todo t positivo, o que permite concluir que

$$
x(t)>0, \quad \text { para todo } t \geq 0 \text {. }
$$

Os dois lemas a seguir serão de grande utilidade para garantir, sob certas condições, a limitação da solução. 
Lema 3.2. Considere a equação

$$
\dot{x}=x f(t, x)
$$

e suponha que f em (3.4) satisfaz as hipóteses (B1)-(B3), onde:

(B1) A função f é contínua e diferenciável com respeito a $x$ em $\mathbb{R}_{+0}^{2}$ e $\frac{\partial f}{\partial x}$ é contínua em $\mathbb{R}_{+0}^{2}$.

(B2) $A$ função $\frac{\partial f}{\partial x} \leq 0$ em $\mathbb{R}_{+0}^{2}$.

(B3) Para constantes $\beta \geq 0$ e $k>0$ tem-se

(i) $f(t, k) \leq \beta$ para $t \geq 0$,

(ii) $\int_{t}^{t+1} f(s, k) d s \leq 0$ para $t \geq 0$.

Dado qualquer $\xi>0$, o problema de valor inicial

$$
\dot{x}=x f(t, x), \quad x(0)=\xi .
$$

tem uma única solução que existe para todo $t \geq 0$. Se $0<\xi \leq k$, então

$$
0<x(t) \leq k e^{\beta} \quad \text { para } \quad t \geq 0,
$$

e se $\xi>k$ existe um $\tau \geq 0$ tal que

$$
0<x(t) \leq \xi e^{\beta} \quad \text { para } \quad t \geq \tau
$$

Prova: A existência local e a unicidade da solução $x(t)$ são garantidas pela hipótese (B1). Como a função nula é uma solução de (3.4), a solução de (3.5) satisfaz $x(t)>0$ em seu intervalo maximal de existência.

Suponha que $\xi>k$. Então, ou $x(t)>k$ para todo $t \geq 0$ para os quais $x(t)$ existe ou existe um $\tau>0$ tal que $x(\tau) \leq k$.

Caso 1: $x(t)>k$ onde a solução existe. Tome $t \geq 0$ e $N$ o inteiro não negativo tal que $N \leq t<N+1$ 
Integrando (3.4) de 0 a $t$, segue que

$$
\int_{0}^{t} \frac{\dot{x}(s)}{x(s)} d s \leq \int_{0}^{t} f(s, x(s)) d s
$$

E, assim,

$$
\ln (x(t))-\ln (x(0)) \leq \int_{0}^{t} f(s, x(s)) d s
$$

$\log \mathrm{O}$

$$
\ln (x(t)) \leq \ln (\xi)+\int_{0}^{t} f(s, x(s)) d s
$$

Portanto,

$$
\begin{aligned}
x(t) & \leq \xi \exp \int_{0}^{t} f(s, x(s)) d s \\
& =\xi \exp \left(\int_{0}^{1} f(s, x(s)) d s+\ldots+\int_{N-1}^{N} f(s, x(s)) d s+\int_{N}^{t} f(s, x(s)) d s\right) \\
& \leq \xi \exp \int_{N}^{t} f(s, x(s)) d s .
\end{aligned}
$$

A função f é decrescente. Logo, $x(s)>k$ implica que $f(s, x(s))<f(s, k)$ e, assim, $\int_{N}^{t} f(s, x(s)) d s<\int_{N}^{t} f(s, k) d s$. Aplicando na desigualdade acima, juntamente com a hipótese $(B 3)(i)$, obtém-se que

$$
x(t) \leq \xi e^{\beta(t-N)} \leq \xi e^{\beta},
$$

pois $t-N \leq 1$.

Portanto, $0<k<x(t) \leq \xi e^{\beta}$ para todo $t \geq 0$ onde $x(t)$ existe. Consequentemente, $x(t)$ existe para todo $t \geq 0$ e (3.7) é obtida tomando $\tau=0$.

Caso 2: existe $\tau>0$ tal que $x(\tau) \leq k$.

Suponha que exista $T>\tau$ tal que $x(T)>k e^{\beta}$. Defina

$$
t_{1}=\sup \{t: x(t)=k, \tau \leq t \leq T\}
$$

Então, $\tau \leq t_{1} \leq T, x\left(t_{1}\right)=k$, e $x(t)>k, t_{1}<t \leq T$. 
Afirmação : $T-t_{1}<1$.

De fato, se $T-t_{1} \geq 1$, então $T>t_{1}+1$ e $x(t)>k$, para $t_{1}<t \leq t_{1}+1$.

Integrando (3.5) de $t_{1}$ a $t_{1}+1$, obtém-se que

$$
x\left(t_{1}+1\right)=x_{t_{1}} e^{\int_{t_{1}}^{t_{1}+1} f(s, x(s) d s)} .
$$

Logo,

$$
k<x\left(t_{1}+1\right)=x\left(t_{1}\right) e^{\int_{t_{1}}^{t_{1}+1} f(s, x(s)) d s} \leq k e^{\int_{t_{1}}^{t_{1}+1} f(s, k) d s} \leq k
$$

fornecendo uma contradição.

Portanto, $T-t_{1}<1$ e, assim,

$$
k e^{\beta}<x(T)=x\left(t_{1}\right) \exp \int_{t_{1}}^{T} f(s, x(s)) d s \leq k \exp \int_{t_{1}}^{T} f(s, k) d s \leq k e^{\beta\left(T-t_{1}\right)} \leq k e^{\beta}
$$

outra contradição. Esse argumento estabelece que não existe tal $T>\tau$ que satisfaça $x(T)>k e^{\beta}$.

Portanto,

$$
x(t) \leq k e^{\beta}<\xi
$$

concluindo a prova de (3.6).

O caso em que $\xi \leq k$. é análogo ao Caso 2, considerando agora $\tau=0$.

Lema 3.3. Suponha que $f$ em (3.4) satisfaz (B1), (B2) e (B4), onde:

(B4) Para constantes $\alpha \geq 0$ e $0<\delta \leq k$,

(i) $f(t, \delta) \geq-\alpha$ para $t \geq 0$,

(ii) $\int_{t}^{t+1} f(s, \delta) d s \geq 0$ para $t \geq 0$.

Se $\xi \geq \delta$, então a solução de (3.5) satisfaz

$$
x(t) \geq \delta e^{-\alpha} \quad \text { para } \quad t \geq 0 .
$$


$E$, se $0<\xi<\delta$, então existe um $\tau \geq 0$ tal que a solução de (3.5) satisfaz

$$
x(t) \geq \xi e^{-\alpha} \quad \text { para } \quad t \geq \tau .
$$

A prova é análoga à do lema anterior.

Teorema 3.1. Assuma que $(A 1)-(A 4)$ estejam satisfeitas e que, para algum $\delta>0$, tem-se $a(t) \geq \delta$ para $t$ suficientemente grande. Então, as soluções $x(\varphi)(t)$ de (3.1), (3.2) estão definidas, são limitadas em $[0, \infty)$ e limitadas inferiormente longe do zero.

Prova: Para $x(t)=x(\varphi)(t), t \geq 0$, a desigualdade

$$
\dot{x}(t) \leq \gamma(t) x(t)
$$

é válida para $\gamma(t)=a(t) b(t)$.

Integrando (3.10) de $t_{0}$ a $t$, com $0 \leq t_{0} \leq t$, segue que

$$
\int_{t_{0}}^{t} \frac{\dot{x}(s)}{x(s)} d s \leq \int_{t_{0}}^{t} \gamma(s) d s
$$

o que implica que

$$
\ln (x(t))-\ln \left(x\left(t_{0}\right)\right) \leq \int_{t_{0}}^{t} \gamma(s) d s .
$$

Logo,

$$
\ln \left(x\left(t_{0}\right)\right) \geq \ln (x(t))-\int_{t_{0}}^{t} \gamma(s) d s .
$$

Portanto,

$$
x\left(t_{0}\right) \geq x(t) e^{-\int_{t_{0}}^{t} \gamma(s) d s}, 0 \leq t_{0} \leq t .
$$

A Observação 1 garante a limitação de $a(t)$. Além disso, (A1) mostra que b também é limitada em $\mathbb{R}$. Sendo assim, $\gamma(t)$ é limitada em $\mathbb{R}$ e é possível tomar $m$ real de tal forma tal que

$$
\sup _{\theta \in[-r, 0]} \int_{t+\theta}^{t} \gamma(s) d s \leq m, t \geq 0 .
$$


Assim (3.11) e (3.12) implicam que $x_{t}(\theta) \geq x(t) e^{-m}$, para $-r \leq \theta \leq 0$. E, de (3.1),

$$
\begin{aligned}
\dot{x}(t) & \leq b(t) x(t)\left[a(t)-L\left(t, x(t) e^{-m}\right)\right]=b(t) x(t)\left[a(t)-L(t, 1) x(t) e^{-m}\right] \\
& =x(t)[\gamma(t)-g(t) x(t)],
\end{aligned}
$$

onde $g(t)=b(t) L(t, 1) e^{-m}$ é uma função contínua e satisfaz:

$$
0<\alpha_{0} \beta_{0} e^{-m} \leq b(t) L(t, 1) e^{-m} \leq \beta^{0} c_{0} e^{-m}
$$

já que as hipóteses (A1) e (A4) estão satisfeitas.

O Lema 3.2 permite concluir que $x$ está definida e é limitada em $[0, \infty)$. Para que ele possa ser aplicado, basta conferir que suas seguintes hipóteses, relativas à $f(t, y)=$ $\gamma(t)-g(t) y$ e $g(t)$ estão satisfeitas.

1. Inicialmente, nota-se que f é contínua, tendo em vista a continuidade das funções a, b e g. Além disso, f é diferenciável com respeito a $y$ e $\frac{\partial f}{\partial y}(t, y)=-g(t)$ é contínua em $\mathbb{R}_{+0}^{2}$.

2. A derivada $\frac{\partial f}{\partial y}(t, y)=-g(t) \leq 0$, pois, $g(t)=b(t) L(t, 1) e^{-m} \geq 0$ para todo $t \geq 0$.

3. Dadas as constantes $\beta \leq 0$ e $k>0$, onde $\beta=\beta^{0} M_{a}$ e $k \geq \frac{M_{a} e^{m} \beta^{0}}{\alpha_{0} \beta_{0}}$, tem-se

$$
f(t, k)=\gamma(t)-g(t) k \leq a(t) \beta^{0}-\alpha_{0} \beta_{0} e^{-m} k \leq a(t) \beta^{0} \leq \beta .
$$

Além disso, $\forall t \geq 0$,

$$
\int_{t}^{t+1} f(s, k) d s=\int_{t}^{t+1}(\gamma(s)-k g(s)) d s \leq \beta^{0} M_{a}-k \alpha_{0} \beta_{0} e^{-m} \leq 0 .
$$

Seja K como na hipótese (A3), isto é, $|a(t)-L(t, \varphi)| \leq K, \quad \forall t \geq 0$. Então, $a(t)-$ $L(t, \varphi) \geq-K, \forall t \geq 0$. Dessa forma, de (3.1) e de (A1) para $t \geq 0$ vale

$$
\dot{x}(t) \geq-b(t) x(t) K \leq-K \beta_{0} x(t) .
$$


Integrando (3.13) de $t+\theta$ a t, $\operatorname{com} \theta \in[-r, 0]$,

$$
\int_{t+\theta}^{t} \frac{\dot{x}(s)}{x(s)} d s \geq-\int_{t+\theta}^{t} K \beta^{0} d s
$$

$\log 0$,

$$
\ln (x(t))-\ln (x(t+\theta)) \geq-K \beta^{0} \theta
$$

permitindo concluir que

$$
x(t+\theta) \leq x(t) e^{-K \beta^{0} \theta} \leq x(t) e^{K \beta^{0} r},
$$

ou seja,

$$
x_{t}(\theta) \leq x(t) e^{\beta^{0} K r}, \quad \text { para } \quad \theta \in[-r, 0] \text { e } t \geq r .
$$

Definindo $h(t)=e^{K r \beta^{0}} b(t) L(t, 1)$ e utilizando (3.1), segue que

$$
\dot{x}(t) \geq x(t)[\gamma(t)-h(t) x(t)]
$$

Além disso, por hipótese, $a(t) \geq \delta$ para t suficientemente grande, o que implica que, $\gamma(t) \geq \delta \beta_{0}>0$. Aplicando o Lema 3.3 conclui-se que $x(t)$ é limitada inferiormente longe do zero.

Teorema 3.2. Assuma que as hipóteses (A1)-(A5) são verdadeiras e que:

(i) $a(t) \rightarrow a_{0}$ quando $t \rightarrow \infty$.

(ii) L em (A5) satisfaz (H2). Então,

$$
\lim _{t \rightarrow \infty} x(t)=x_{*}
$$

onde $x(t)=x(\varphi)(t)$ é a solução do PVI (3.1), (3.2) e $x_{*}=\frac{a_{0}}{\|L\|}$

Prova: Sendo $x(t)$ solução de (3.1), (3.2) e, através da mudança de variáveis $y(t)=$ $\frac{x(t)-x_{*}}{x_{*}}, y(t)$ é solução de (3.3). A demonstração pode ser dividida em dois casos: 
Caso 1: Suponha que $y(t)$ seja eventualmente monótona, isto é, que exista $T \in \mathbb{R}$ tal que $y(t)$ seja monótona quando $t \geq T$.

O Teorema 3.1 afirma que $y(t)$ é limitada em $[0, \infty)$ e limitada inferiormente longe de zero. Dessa forma, a monotonicidade eventual de y garante a existência de c tal que

$$
\lim _{t \rightarrow \infty} y(t)=c
$$

Caso 1.1: Suponha que $c>0$.

Fixe $\epsilon>0$ suficientemente pequeno de tal maneira que

$$
c-\epsilon>0 \quad \text { e } \epsilon+a_{0}[\epsilon-(c-\epsilon)(1-\epsilon)]<0 .
$$

As hipóteses (i), (A5) e (3.15) garantem que existe $T_{1}$ de tal forma que

$$
\begin{gathered}
y(t) \geq c-\epsilon, \quad \forall t \geq T_{1}-r \\
a(t) \leq a_{0}+\epsilon \quad \text { e } \quad L(t, 1) \geq\|L\|(1-\epsilon), \quad \forall t \geq T_{1}
\end{gathered}
$$

Dessa forma, para $t \geq T_{1}$, integrando (3.1) de $T_{1}$ a t:

$$
\int_{T_{1}}^{t} \frac{\dot{y}(s)}{y(s)+1} d s=\int_{T_{1}}^{t} b(s)\left[a(s)-x_{*} L\left(s, y_{s}\right)-x_{*} L(s, 1)\right] d s
$$

$\log 0$

$$
\ln (y(t)+1)-\ln \left(y\left(T_{1}\right)+1\right)=\int_{T_{1}}^{t} b(s)\left[a(s)-x_{*} L\left(s, y_{s}\right)-x_{*} L(s, 1)\right] d s,
$$

mostrando que

$$
\begin{aligned}
y(t)+1 & \leq\left(y\left(T_{1}\right)+1\right) e^{\int_{T_{1}}^{t} b(s)\left[a(s)-x_{*} L\left(s, y_{s}\right)-x_{*} L(s, 1)\right] d s} \\
& \leq\left(y\left(T_{1}\right)+1\right) e^{\beta_{0}\left[\epsilon+a_{0}[\epsilon-(c-\epsilon)(1-\epsilon)]\left(t-T_{1}\right)\right.}, \quad t \geq T_{1} .
\end{aligned}
$$

Fazendo $t \rightarrow \infty$, conclui-se que $c \leq-1$, o que contraria o fato de que $c>0$.

Caso 1.2: Ao supor-se $c<0$, chega-se a uma contradição de maneira análoga à do Caso 1.1. 
Portanto $c=0$ e o resultado está provado.

Caso 2: Suponha que $y(t)$ não seja eventualmente monótona. Defina

$$
\liminf _{t \rightarrow \infty} y(t)=-v \quad \limsup _{t \rightarrow \infty} y(t)=u
$$

O Teorema 3.1 afirma que y é limitada em $[0, \infty)$ e limitada inferiormente longe de zero, $\log 0-1<-v \leq u<\infty$.

Fixe $\varepsilon>0$ e considere $T>0$ tal que

$$
-v-\varepsilon \leq y(t) \leq u+\varepsilon, t>T
$$

Para concluir a prova do teorema, basta mostrar que $u=v=0$. Para isso, suponha que $|v| \leq u$ e tome $\left(t_{n}\right) \subset \mathbb{R}$ uma sequência tal que

$$
t_{n}-r>T, \quad y^{\prime}\left(t_{n}\right)=0, \quad y\left(t_{n}\right)>0, \quad \lim _{n \rightarrow \infty} y\left(t_{n}\right)=u \quad e \quad \lim _{n \rightarrow \infty} t_{n}=\infty
$$

\section{Então}

$$
y^{\prime}\left(t_{n}\right)=b\left(t_{n}\right)\left(y\left(t_{n}\right)+1\right)\left[a\left(t_{n}\right)-x_{*} L\left(t_{n}, y_{t_{n}}\right)-x_{*} L\left(t_{n}, 1\right)\right]=0,
$$

$\log 0$

$$
a\left(t_{n}\right)-x_{*} L\left(t_{n}, y_{t_{n}}\right)-x_{*} L\left(t_{n}, 1\right)=0
$$

As funções $y$ e $\dot{y}$ são limitadas. Sendo assim, $y$ é Lipschitz e, portanto, $y_{t_{n}}$ é equicontínua e uniformemente limitada. O Teorema de Ascoli garante que $y_{t_{n}}$ possui subsequência, aqui representada com a mesma notação, de tal forma que $y_{t_{n}} \rightarrow \varphi$, para alguma $\varphi \in C$.

Como $y\left(t_{n}\right) \rightarrow u$, pela unicidade do limite, segue que $\varphi(0)=u$. Por outro lado,

$$
\begin{aligned}
0 & =a\left(t_{n}\right)-x_{*} L\left(t_{n}, y_{t_{n}}\right)-x_{*} L\left(t_{n}, 1\right) \\
& =a\left(t_{n}\right)-x_{*} L\left(t_{n}, y_{t_{n}}\right)-x_{*} L\left(t_{n}, 1\right)-x_{*} L\left(t_{n}, \varphi\right)+x_{*} L\left(t_{n}, \varphi\right) \\
& =\left[a\left(t_{n}\right)-x_{*} L\left(t_{n}, 1\right)\right]-x_{*}\left[L\left(t_{n}, y_{t_{n}}\right)-L\left(t_{n}, \varphi\right)\right]-x_{*} L\left(t_{n}, \varphi\right) .
\end{aligned}
$$


Fazendo $n \rightarrow \infty$, segue que $L(\varphi)=0$; pois $a\left(t_{n}\right) \rightarrow a_{0}, L\left(t_{n}, \varphi\right) \rightarrow L(\varphi), L\left(t_{n}, 1\right) \rightarrow$ $L(1)=\|L\|$ e $x_{*}=\frac{a_{0}}{\|L\|}$.

De (3.17) e da hipótese $|v| \leq u$, segue que

$$
\left|y_{t_{n}}\right|_{C}=\sup _{s \in[0, \infty)}\left|y_{t_{n}}(s)\right| \leq u+\varepsilon
$$

Fazendo $n \rightarrow \infty$ obtém-se:

$$
\left|y_{t_{n}}\right|_{C} \rightarrow|\varphi|_{C} \leq u+\varepsilon
$$

e, como $\varepsilon$ é arbitrário, conclui-se que

$$
|\varphi|_{C} \leq u=\varphi(0)
$$

Da hipótese (H2), $u=|\varphi|_{C}=0$, o que implica que $v=0$.

Para o caso em que $|u| \leq v$, a demonstração segue da mesma forma. Basta agora considerar $\left(s_{n}\right) \subset \mathbb{R}$ uma sequência tal que

$$
s_{n}-r>T, \quad y^{\prime}\left(s_{n}\right)=0, \quad y\left(s_{n}\right)<0, \quad \lim _{n \rightarrow \infty} y\left(s_{n}\right)=-v \quad e \quad \lim _{n \rightarrow \infty} s_{n}=\infty .
$$

O corolário a seguir pode ser visto como um caso particular de (3.1), sob as hipóteses (A1)-(A5).

Corolário 3.2.1. Considere a equação diferencial funcional:

$$
\dot{x}(t)=x(t)\left[a(t)-h(t) S\left(x_{t}\right)\right]
$$

onde $a, h: \mathbb{R} \rightarrow \mathbb{R}$ são funções contínuas, $L: C \rightarrow \mathbb{R}$ é um operador linear positivo não nulo e existem constantes $\alpha_{0}, \alpha^{0}, \beta_{0}$ e $\beta^{0}$ tais que

$$
\alpha_{0} \leq a(t) \leq \alpha^{0}, \quad \text { e } \quad 0<\beta_{0} \leq h(t) \leq \beta^{0}, \forall t \in \mathbb{R}
$$


Então, todas as soluções $x(\varphi)(t)$ do P.V.I. (3.18), (3.2) são limitadas em $[0, \infty)$ e limitadas inferiormente longe do zero. Além disso, se

$$
\lim _{t \rightarrow \infty} a(t)=a_{0}, \quad \lim _{t \rightarrow \infty} h(t)=h_{0}
$$

e L satisfaz (H2), então

$$
\lim _{t \rightarrow \infty} x(\varphi)(t)=x_{*}
$$

onde $x_{*}=\frac{a_{0}}{h_{0}\|S\|}$. 

Capítulo

4

\section{Equação autônoma com linearidade não positiva}

Este capítulo aborda um caso mais geral da equação (2.1), na qual o operador $L$ : $C \rightarrow \mathbb{R}$ é linear, não nulo e limitado, mas não necessariamente positivo. Para esse tipo de equação, será desenvolvido um importante teorema visando garantir a estabilidade assintótica, o qual poderá ser aplicado em casos em que não há a possibilidade de se utilizar técnicas usualmente empregadas, tal como o Método de Liapunov.

\subsection{Equação Logística Retardada com uma linearidade autônoma não positiva}

Considere a equação $(2.1)$ e $\eta:[-r, 0] \rightarrow \mathbb{R}$ uma função de variação limitada de tal forma que, para $\varphi \in C$,

$$
L(\varphi)=\int_{-r}^{0} \varphi(\theta) d \eta(\theta)
$$

Dessa forma, é possível decompor $\eta=\eta_{1}-\eta_{2}$, onde as funções $\eta_{i}:[-r, 0] \rightarrow \mathbb{R}$, $i=1,2$, são não decrescentes, o que é sempre possível já que $\eta$ é de variação limitada. 
Logo, L pode ser visto como:

$$
L(\varphi)=L_{1}(\varphi)-L_{2}(\varphi), \quad \varphi \in C
$$

onde os operadores $L_{1}$ e $L_{2}$ são lineares, positivos e da forma

$$
L_{i}(\varphi)=\int_{-r}^{0} \varphi(\theta) d \eta_{i}(\theta), i=1,2
$$

A partir daí, a equação (2.1) se torna da forma:

$$
\dot{x}(t)=b(t) x(t)\left[1-L_{1}\left(x_{t}\right)+L_{2}\left(x_{t}\right)\right] .
$$

Note que (4.1) tem um equilíbrio positivo dado por

$$
x_{*}=\frac{1}{\left\|L_{1}\right\|-\left\|L_{2}\right\|} \Leftrightarrow\left\|L_{1}\right\|>\left\|L_{2}\right\|
$$

Além disso, bem como nos capítulos anteriores, serão consideradas condições iniciais admissíveis

$$
x_{0}=\varphi \quad \text { tal que } \varphi(\theta) \geq 0, \theta \in[-r, 0) \quad \text { e } \quad \varphi(0)>0,
$$

e as hipóteses

(H1) (i) $b: \mathbb{R} \rightarrow \mathbb{R}$ é uma função contínua e existem constantes $\beta_{0}$ e $\beta^{0}$ tais que $0<\beta_{0} \leq b(t) \leq \beta^{0}, \forall t \in \mathbb{R}$.

(ii) $L: C \rightarrow \mathbb{R}$ é um operador linear positivo $\operatorname{com} l:=\|L\|>0$.

(H2) Para toda $\varphi \in C$ tal que $|\varphi|_{C}=\varphi(0)>0$, segue que $L(\varphi)>0$.

$\left(\mathrm{H} 2^{*}\right)$ Existe uma constante $d \in(0,1)$ tal que se $\varphi \in C, \varphi(0)>0$ e $L(\varphi)=0$, então $\varphi(0) \leq d|\varphi|_{C}$

Observação 3. Quando a hipótese (H2) for satisfeita, necessariamente tem-se $\left\|L_{1}\right\|>$ $\left\|L_{2}\right\|$. De fato, tomando $\varphi \in C$ tal que $\varphi \equiv 1$, segue que $|\varphi|_{C}=1=\varphi(0)>0$. Assim, de 
(H2), $L(\varphi \equiv 1)>0$. Logo,

$$
L(1)=\int_{-r}^{0} d \eta_{1}(\theta)-\int_{-r}^{0} d \eta_{2}(\theta)=\left\|L_{1}\right\|-\left\|L_{2}\right\|>0
$$

Consequentemente, a solução $x(\varphi)(t)$ de (4.1), (4.2) é positiva para $t \geq 0$ no intervalo maximal de existência.

\subsection{Resultados importantes}

Teorema 4.1. Considere $L=L_{1}-L_{2}$, onde $L_{1}$ e $L_{2}$ são operadores lineares positivos. Assuma que as hipóteses (H1)(i) e (H2) estejam satisfeitas. Então, as soluções $x(t)=$ $x(\varphi)(t)$ de (4.1) estão definidas, são limitadas em $[0, \infty)$ e são limitadas inferiormente longe do zero. Mais ainda,

$$
\lim _{t \rightarrow \infty} x(t)=x_{*}
$$

onde $x_{*}=\frac{1}{\left\|L_{1}\right\|-\left\|L_{2}\right\|}$ é o único equilíbrio positivo de (4.1).

Prova: Tome $x(t)=x(\varphi)(t)$ solução do P.V.I. (4.1), (4.2) definida em $I=[-r, a)$, onde $a \in \mathbb{R}$ ou $a=+\infty$. Fixe $t_{0} \in[0, a)$ e defina $k=\max _{-r \leq t \leq t_{0}}\left|x(t)-x_{*}\right|$. Dessa forma,

$$
\left|x(t)-x_{*}\right| \leq k, \forall t \in\left[-r, t_{0}\right]
$$

Pelo Lema 4.5, segue que $\left|x(t)-x_{*}\right| \leq k, \forall t \in I$.

Suponha que exista $t_{1} \in\left(t_{0}, a\right)$ tal que $\left|x\left(t_{1}\right)-x_{*}\right|>k$. Defina

$$
T=\min \left\{t \in\left[t_{0}, t_{1}\right]:\left|x(t)-x_{*}\right|=\sup _{t_{0} \leq s \leq t_{1}}\left|x(s)-x_{*}\right|\right\}
$$

Assim, $T \in\left(t_{0}, t_{1}\right] \mathrm{e}$

$$
\left|x(s)-x_{*}\right|<\left|x(T)-x_{*}\right|, \forall s \in\left(t_{0}, T\right) .
$$

Dessa forma, de (4.3) e (4.4) segue que $\left|x(s)-x_{*}\right| \leq k<\left|x(T)-x_{*}\right|, \forall s \in\left[-r, t_{0}\right]$. 
Logo,

$$
\left|x(s)-x_{*}\right|<\left|x(T)-x_{*}\right|, \forall s \in[-r, T) .
$$

Se $\left|x(T)-x_{*}\right|>0$, então $\left|x(s)-x_{*}\right|<x(T)-x_{*}, \forall s \in[-r, T)$. Logo, $x(s)-x_{*}<$ $x(T)-x_{*}, \forall s \in[-r, T)$, mostrando que $x(s)<x(T), \forall s \in[-r, T)$. Portanto, $\dot{x}(T) \geq 0, \mathrm{o}$ que contradiz a hipótese (H2), pois (H2) implica que $L\left(x_{T}-x_{*}\right)=L_{1}\left(x_{T}-x_{*}\right)-L_{2}\left(x_{T}-\right.$ $\left.\left.x_{*}\right)\right]>0$, já que $x(T)-x_{*}>0$. Logo,

$$
\dot{x}(T)=-b(T) x(T)\left[L_{1}\left(x_{T}-x_{*}\right)-L_{2}\left(x_{T}-x_{*}\right)\right]<0 .
$$

Para o caso em que $\left|x(T)-x_{*}\right|<0$, segue que $\left|x(s)-x_{*}\right|<x_{*}-x(T), \forall s \in[-r, T)$. Logo, $x_{*}-x(s)<x_{*}-x(T), \forall s \in[-r, T)$, o que implica que $x(s)>x(T), \forall s \in[-r, T)$, mostrando que $\dot{x}(T) \leq 0$. Por outro lado, (H2) implica que $L\left(x_{T}-x_{*}\right)=L_{1}\left(x_{T}-x_{*}\right)-$ $\left.L_{2}\left(x_{T}-x_{*}\right)\right]<0$, já que $x(T)-x_{*}<0$. Logo,

$$
\dot{x}(T)=-b(T) x(T)\left[L_{1}\left(x_{T}-x_{*}\right)-L_{2}\left(x_{T}-x_{*}\right)\right]>0,
$$

fornecendo uma contradição.

Então conclui-se que não existe tal $t_{1}$, ou seja,

$$
\left|x(T)-x_{*}\right| \leq k, \forall t \in I
$$

Sendo assim, obtém-se que $x(t)$ está definida para todo $t \geq 0$, é limitada em todo o seu domínio e é limitada inferiormente longe do zero.

Para finalizar a demonstração, falta provar que

$$
\lim _{t \rightarrow \infty} x(t)=x_{*} .
$$

Para isso, considere a troca de variáveis $y=\frac{x-x_{*}}{x_{*}}$.

Então, $y(t)$ é solução de

$$
\dot{y}(t)=-x_{*} b(t)(1+y(t))\left[L_{1}\left(y_{t}\right)-L_{2}\left(y_{t}\right)\right] .
$$


Caso 1: a função $y(t)$ é eventualmente monótona.

Considere $c=\lim _{t \rightarrow \infty} y(t)$.

Suponha que $c>0$. Fixe $\varepsilon>0$ tal que

$$
\varepsilon<\frac{c\left(\left\|L_{1}\right\|-\left\|L_{2}\right\|\right)}{\left\|L_{1}\right\|+\left\|L_{2}\right\|} \leq c
$$

e tome $T_{1}$ tal que

$$
c-\varepsilon \leq y_{t} \leq c+\varepsilon, \forall t \geq T_{1}
$$

Integrando (4.5) de $T_{1}$ a $t$, obtém-se que:

$$
\int_{T_{1}}^{t} \frac{\dot{y}(\tau)}{1+y(\tau)} d(\tau)=-\int_{T_{1}}^{t} x_{*} b(\tau)\left[L_{1}\left(y_{\tau}\right)-L_{2}\left(y_{\tau}\right)\right] d(\tau)
$$

Assim,

$$
\ln (1+y(t))-\ln \left(1+y\left(T_{1}\right)\right)=-\int_{T_{1}}^{t} x_{*} b(\tau)\left[L_{1}\left(y_{\tau}\right)-L_{2}\left(y_{\tau}\right)\right] d(\tau)
$$

Logo,

$$
y(t)=\left(1+y\left(T_{1}\right)\right) e^{-\int_{T_{1}}^{t} x_{*} b(\tau)\left[L_{1}\left(y_{\tau}\right)-L_{2}\left(y_{\tau}\right)\right] d(\tau)} .
$$

Para $t \geq T_{1}$, de (4.6) e (4.7), segue que:

$$
L_{1}\left(y_{t}\right)-L_{2}\left(y_{t}\right) \geq(c-\varepsilon)\left\|L_{1}\right\|-(c+\varepsilon)\left\|L_{2}\right\|=c\left(\left\|L_{1}\right\|-\left\|L_{2}\right\|\right)-\varepsilon\left(\left\|L_{1}\right\|+\left\|L_{2}\right\|\right)>0 .
$$

Sendo assim, para $t \geq T_{1}$, vale:

$$
y(t) \leq\left(1+y\left(T_{1}\right)\right) e^{-x_{*} \beta_{0}\left(t-T_{1}\right)\left[c\left(\left\|L_{1}\right\|-\left\|L_{2}\right\|\right)-\varepsilon\left(\left\|L_{1}\right\|+\left\|L_{2}\right\|\right)\right]}-1 .
$$

Fazendo $t \rightarrow \infty$, conclui-se que $c \leq-1$, contrariando a hipótese $c>0$.

Ao supor-se que $c<0$, procedendo de maneira análoga, chega-se também a uma contradição. Dessa forma, pode-se concluir que $c=0$.

Caso 2: $y(t)$ não é eventualmente monótona. 
Defina

$$
\liminf _{t \rightarrow \infty} y(t)=-v \quad e \quad \limsup _{t \rightarrow \infty} y(t)=u .
$$

Então, $0 \leq v<1$ e $0 \leq u<\infty$. Note que a prova estará finalizada ao mostrar-se que $u=v=0$.

Fixe $\varepsilon>0$ e considere $T>0$ tal que

$$
-v-\varepsilon \leq y(t) \leq u+\varepsilon, \quad \text { para } t>T
$$

Caso 2.1: $v \leq u$.

Considere $\left(t_{n}\right) \subset \mathbb{R}$ uma sequência tal que

$$
t_{n}-r>T, \quad y^{\prime}\left(t_{n}\right)=0, \quad y\left(t_{n}\right)>0, \quad \lim _{n \rightarrow \infty} y\left(t_{n}\right)=u \quad e \quad \lim _{n \rightarrow \infty} t_{n}=\infty
$$

Então, de (4.5) segue que:

$$
0=\dot{y}\left(t_{n}\right)=-x_{*} b\left(t_{n}\right)\left(1+y\left(t_{n}\right)\right)\left[L_{1}\left(y_{t_{n}}\right)-L_{2}\left(y_{t_{n}}\right)\right] .
$$

Logo,

$$
L_{1}\left(y_{t_{n}}\right)-L_{2}\left(y_{t_{n}}\right)=0 \text {. }
$$

Segundo o Teorema 4.1 sabe-se que y é limitada. Além disso, de (4.5) percebe-se que $\dot{y}$ é limitada. Logo, $y$ é Lipschitz. Mais ainda, $y_{t_{n}}$ é equicontínua e uniformemente limitada. Sendo assim, o Teorema de Ascoli garante que $y_{t_{n}}$ possui uma subsequência, aqui representada com a mesma notação, tal que $y_{t_{n}} \rightarrow \varphi$, para alguma $\varphi \in C$.

De acordo com (4.9), $L(\varphi)=0$. Por outro lado, analisandos-e (4.8) juntamente com o fato de que $v \leq u$, obtém-se que: $|y(t)| \leq u+\varepsilon, \forall t>T$. Logo, $\left|y_{t_{n}}\right|_{C}=$ $\sup _{s \in[0, \infty)}\left|y\left(t_{n}+s\right)\right| \leq u+\varepsilon$.

Fazendo $\varepsilon \rightarrow \infty$ e, como $\varepsilon>0$ é arbitrário, obtém-se que

$$
|\varphi|_{C} \leq u=\varphi(0)
$$

Conclui-se que $u=0$, pois, como $L(\varphi)=0,(\mathrm{H} 2)$ implica que $|\varphi|_{C}=\varphi(0)=0$. Assim, 
$v=0$.

Caso 2.2: $v \geq u$.

A prova é análoga à do Caso 2.1. Basta considerar uma sequência $\left(s_{n}\right) \subset \mathbb{R}$ satisfazendo:

$$
s_{n}-r>T, \quad y^{\prime}\left(s_{n}\right)=0, \quad y\left(s_{n}\right)<0, \quad \lim _{n \rightarrow \infty} y\left(s_{n}\right)=-v \quad e \quad \lim _{n \rightarrow \infty} s_{n}=\infty .
$$

Teorema 4.2. Assuma que (H1)(i) esteja satisfeita. Tome $\varphi \in C$ e considere

$$
L(\varphi)=b_{0} \varphi(0)+\widetilde{L}_{1}(\varphi)-L_{2}(\varphi)
$$

onde $b_{0}>0, \widetilde{L}_{1}$ e $L_{2}$ são operadores lineares positivos.

Se $b_{0}>\left\|L_{2}\right\|$, então a solução de (4.1), (4.2) está definida, é limitada para $t \geq 0$ e é limitada inferiormente longe do zero.

Prova : Considere $x(t)=x(\varphi)(t)$ solução de (4.1), (4.2) definida em $I=[-r, a)$, onde $a \in \mathbb{R}$ ou $a=+\infty$.

Afirmação : $x(t)<M$, para todo $t \in I$, onde

$$
M<\max \left[|\varphi|_{C},\left(b_{0}-\left\|L_{2}\right\|\right)^{-1}\right] .
$$

De fato, suponha que exista $T_{1}>0$ tal que

$$
0 \leq x(t)<M, \forall t \in\left[-r, T_{1}\right] \quad e \quad x\left(T_{1}\right)=M .
$$

Então, $\dot{x}\left(T_{1}\right) \geq 0$. Mas, de (4.1), segue que

$$
\begin{aligned}
\dot{x}\left(T_{1}\right) & =b\left(T_{1}\right) x\left(T_{1}\right)\left[1-L\left(x_{T_{1}}\right)\right]=b\left(T_{1}\right) x\left(T_{1}\right)\left[1-b_{0} x\left(T_{1}\right)-\widetilde{L}_{1}\left(x_{T_{1}}\right)+L_{2}\left(x_{T_{1}}\right)\right] \\
& \left.\leq b\left(T_{1}\right) x\left(T_{1}\right)\left[1-b_{0} x\left(T_{1}\right)+L_{2}\left(x_{T_{1}}\right)\right] \leq b\left(T_{1}\right) x\left(T_{1}\right)\left[1-b_{0} M+\left\|L_{2}\right\| M\right]\right] \\
& =b\left(T_{1}\right) x\left(T_{1}\right)\left[1-M\left(b_{0}-\left\|L_{2}\right\|\right)\right]<0,
\end{aligned}
$$


gerando uma contradição.

Assim, conclui-se que $x(t)$ está definida e é limitada para $t \geq 0$. Além disso, $x(t)$ é limitada inferiormente longe do zero.

Corolário 4.2.1. Assuma que a hipótese (H1)(i) esteja satisfeita e considere L como em (4.10), onde $b_{0}>0, \widetilde{L}_{1}$ e $L_{2}$ são operadores lineares positivos. Se (H2) estiver satisfeita, então a solução do P.V.I. (4.1), (4.2) está definida, é limitada para $t \geq 0$ e é limitada inferiormente longe do zero. Além disso, satisfaz

$$
\lim _{t \rightarrow \infty} x(t)=x_{*}
$$

onde $x_{*}=\left(b_{0}+\left\|\tilde{L_{1}}\right\|-\left\|L_{2}\right\|\right)^{-1}$.

\subsection{Aplicação}

Para finalizar, vamos considerar a seguinte aplicação.

Aplicação 4.1. Considere a equação escalar Lotka-Volterra com retardos distribuídos:

$$
\dot{x}(t)=\beta(t) x(t)\left[\gamma-a x(t)+b \int_{-r}^{0} x(t+\theta) d \mu_{1}(\theta)-c \int_{-r}^{0} x(t+\theta) d \mu_{2}(\theta)\right],
$$

onde a função $\beta: \mathbb{R} \rightarrow \mathbb{R}$ é contínua e existem constantes $\beta_{0}$, $\beta^{0}$ tais que $0<\beta_{0} \leq \beta(t) \leq$ $\beta^{0}$, para todo $t \in \mathbb{R}$. Além disso, $\gamma$, a, b e c são constantes não negativas, as funções $\mu_{i}$ são não decrescentes e $\int_{-r}^{0} d \mu_{i}(\theta)=1, i=1,2$.

Considere o operador

$$
L(\varphi)=a \varphi(0)+L_{0}(\varphi)
$$

com

$$
L_{0}(\varphi)=c \int_{-r}^{0} \varphi(\theta) d \mu_{2}(\theta)-b \int_{-r}^{0} \varphi(\theta) d \mu_{1}(\theta)
$$

Pelo Corolário 4.3, se L satisfaz (H2), então todas as soluções de (4.10), com condições iniciais admissiveis, estão definidas para $t \geq 0$ e convergem para $x_{*}=\frac{\gamma}{a+c-b}$. 
Para o caso em que $\beta(t) \equiv 1$, Kuang (1993) demonstrou, utilizando o Método de Liapunov com as hipóteses,

$$
b+c \leq a, \quad b-c<1,
$$

a estabilidade assintótica global do equilíbrio positivo $x_{*}$ através do teorema:

Teorema 4.1. A equação

$$
\dot{x}(t)=x(t)\left[\gamma-a x(t)+b \int_{-r}^{0} x(t+\theta) d \mu_{1}(\theta)-c \int_{-r}^{0} x(t+\theta) d \mu_{2}(\theta)\right]
$$

é estável assintotica e globalmente para todo $\mu_{i}(\theta), i=1,2$ e para todas condições iniciais $\phi$ satisfazendo $\phi(\theta)>0, \phi \in C$, se

$$
b+c \leq a \quad e \quad b-c<a .
$$

Ou seja,

$$
\lim _{t \rightarrow \infty} x(\phi)(t)=\frac{\gamma}{a+c-b}
$$

Note que, se $c=0$ e $b<a$, o teorema acima é satisfeito. E o mesmo resultado pode ser obtido utilizando o Corolário 4.3. Basta mostrar que L satisfaz (H2). De fato,

$$
L(\varphi)=a \varphi(0)-b \int_{-r}^{0} \varphi(\theta) d \mu_{1}(\theta) \geq a \varphi(0)-b \varphi(0)=\varphi(0)(a-b)>0 .
$$

Quando c $>0$, as hipóteses do Teorema 4.4 estão satisfeitas e a função $\mu_{2}$ é tal que existe $\varepsilon \in(0, r)$ satisfazendo $\mu_{2}(\theta)<\mu_{2}(0)$, para todo $-\varepsilon<\theta<0$. Entãa, a condição $b+c \leq a$ implica na hipótese (H2). De fato, seja $\varphi \in C$ tal que $|\varphi|_{C}=\varphi(0)>0$. Para mostrar que $L(\varphi)>0$, considere os dois casos:

Caso 1: $b+c<a$. Dessa forma,

$$
\begin{aligned}
L(\varphi) & =a_{0} \varphi(0)+c \int_{-r}^{0} \varphi(\theta) d \mu_{2}(\theta)-b \int_{-r}^{0} \varphi(\theta) d \mu_{1}(\theta) \\
& \geq a_{0} \varphi(0)-c \int_{-r}^{0} \varphi(\theta) d \mu_{2}(\theta)-b \int_{-r}^{0} \varphi(\theta) d \mu_{1}(\theta) \\
& \geq a_{0} \varphi(0)-(c+b)|\varphi|_{C}>0 .
\end{aligned}
$$


Caso 2: $b+c=a$.

$$
\begin{aligned}
L(\varphi) & =(b+c) \varphi(0)+c \int_{-r}^{0} \varphi(\theta) d \mu_{2}(\theta)-b \int_{-r}^{0} \varphi(\theta) d \mu_{1}(\theta) \\
& =c \varphi(0)+c \int_{-r}^{0} \varphi(\theta) d \mu_{2}(\theta)+b \int_{-r}^{0}[\varphi(0)-\varphi(\theta)] d \mu_{1}(\theta) \\
& \geq c \varphi(0)+c \int_{-r}^{0} \varphi(\theta) d \mu_{2}(\theta) .
\end{aligned}
$$

Escolha $\xi \in(-\varepsilon, 0)$ tal que $\varphi(\theta)>0$, para $\theta \in[\xi, 0]$. Sendo assim,

$$
\begin{aligned}
L(\varphi) & \geq c \varphi(0)+c \int_{-r}^{0} \varphi(\theta) d \mu_{2}(\theta) \geq c \varphi(0)+c \int_{-r}^{\xi} \varphi(\theta) d \mu_{2}(\theta) \\
& \geq c \varphi(0)-c \varphi(0)\left(\mu_{2}(\xi)-\mu_{2}(-r)\right)>c \varphi(0)\left(1-\left\|L_{2}\right\|\right)=0 .
\end{aligned}
$$

Considere agora o caso em que $c>0$ e $\left\|L_{0}\right\|<a<b+c$.

Note que

$$
\left|L_{0}(\varphi)\right|=\left[c \int_{-r}^{0} \varphi(\theta) d \mu_{2}(\theta)-b \int_{-r}^{0} \varphi(\theta) d \mu_{1}(\theta)\right] \leq c|\varphi|_{C}+b|\varphi|_{C}=|\varphi|_{C}(c+b),
$$

ou seja,

$$
\left\|L_{0}\right\| \leq c+b
$$

Nesse caso, o Teorema 4.4 não se aplica. Entretanto, o operador L satisfaz a hipótese (H2*), logo, as hipóteses do Corolário 4.3 estão satisfeitas e, portanto, a estabilidade assintótica global está garantida.

Para mostrar que $\left(H^{*}\right)$ realmente está satisfeita, tome $\varphi \in C$ tal que $\varphi(0)>0$ e $L(\varphi)=0$. Então,

$$
0=L(\varphi)=a_{0} \varphi(0)+L_{0}(\varphi) \geq a_{0} \varphi(0)-\left|L_{0}(\varphi)\right| \geq a_{0} \varphi(0)-\left\|L_{0}\right\||\varphi|_{C},
$$

ou seja,

$$
\left\|L_{0}\right\||\varphi|_{C} \geq a_{0} \varphi(0)
$$


$E$, portanto, tomando-se $d=\frac{\left\|L_{0}\right\|}{a_{0}} \in(0,1)$, obtém-se que

$$
\varphi(0) \leq \frac{\left\|L_{0}\right\|}{a_{0}}|\varphi|_{C}=d|\varphi|_{C}
$$

Dessa forma, a conclusão é que, nos casos em que é possivel aplicar o Teorema 4.4, o mesmo resultado pode ser obtido através do Corolário 4.3 Entretanto, há casos em que o Teorema 4.4 não pode ser utilizado, mas a estabilidade assintótica do equilíbrio positivo é garantida aplicando-se o Corolário 4.3. 


\section{Referências Bibliográficas}

[1] J. K. Hale e S. M. V. Lunel; Introduction to Functional Differential Equations; Springer-Verlag, 1993.

[2] J. W.-H So e J. S. Yu.; Global attractivity for a population model with time delay; Proc. Am. Math. Soc. 123 (1995), 2687-2694.

[3] J. W.-H So e J. S. Yu.; Global stability for a general population model with time delays; Fields Inst. Commun. 21 (1999), 447-457.

[4] Proceedings of the Royal Society of Edinburgh, 133A,1057-1073,

[5] R. R. Vance e E. A. Coddington; A nonautonomous model of population growth; J. Math. Biol. 27 (1989), 491-506.

[6] T. Faria e E. Liz; Boundedness and asymptotic stability for delayed equations of logistic type; 2003.

[7] Y. Kuang; Global stability for a class of nonlinear nonautonomous delay equations; Nonlin. Analysis 17 (1991), 627-634.

[8] Y. Kuang; Delay differential equations with applications in population dynamics; (Academic, 1993). 